\title{
Engineering microfluidic concentration gradient generators for biological applications
}

\author{
Alicia G. G. Toh, ${ }^{1,2}$ Z.P. Wang, ${ }^{2}$ Chun Yang, ${ }^{1}$ and Nam-Trung Nguyen ${ }^{3 *}$ \\ 1 School of Mechanical and Aerospace Engineering, Nanyang Technological University, 50 \\ Nanyang Avenue, Singapore 639798 \\ 2 Singapore Institute of Manufacturing Technology, 71 Nanyang Drive, Singapore 63807 \\ 3 Queensland Micro and Nanotechnology Centre, Griffith University, Brisbane, 4111, Australia \\ *Email: nam-trung.nguyen@griffith.edu.au
}

\begin{abstract}
This paper reviews the latest developments in the design and fabrication of concentration gradient generators for microfluidics- based biological applications. New gradient generator designs and their underlying mass transport principles are discussed. The review provides a blueprint for design considerations of concentration gradients in different applications, specifically biological studies. The paper discusses the basic phenomena associated with microfluidic gradient generation and the different gradient generation modes used in static and dynamic biological assays. Finally, the paper summarizes all factors to consider when using concentration gradient generators and puts forward perspectives on the future development of these devices.
\end{abstract}

\section{Introduction}

Within the human body, concentration gradients of biomolecules are regulated to control many basic cell functions. Biological processes such as development (Chung, Flanagan et al. 2005; Park, Hwang et al. 2007; Gupta, Kim et al. 2010), immune response (Lin, Nguyen et al. 2004; Li, Liu et al. 2008; Wang, Li et al. 2008; Kothapalli, van Veen et al. 2011), wound healing (Lin 2009; Meer, Vermeul et al. 2010), embryogenesis (Heo, Cabrera et al. 2010) and cancer metastasis (Wang, Saadi et al. 2004; Saadi, Wang et al. 2006; Mosadegh, Saadi et al. 2008; Nandagopal, Wu et al. 2011) depend on biomolecular gradients to augment and regulate cell signalling pathways. To understand the influence of chemical stimuli on the signaling pathways in cells, biologists have sought alternative methods to artificially engineer in-vivo cell microenvironments. Early work in this field relied on in-vitro platforms such as the Boyden chamber (Boyden 1962), Dunn slide chamber (Zicha, Dunn et al. 1991), Zigmond chamber (Zigmond and Hirsch 1973) etc. A more recent adoption in the field of cellular signaling analysis, uses the agarose/petri dish (Kim, Kim et al. 2010) and the micro-aspirator to recreate in-vivo concentration gradients of biomolecules at the single cell level. Although these platforms have generated much knowledge on 
how cells respond through intra- and extracellular signalling pathways, a limitation of existing platforms has been the inability to reach the length scales that are actually relevant to biological cells (El-Ali, Sorger et al. 2006). Most cell diameters range from 1 to $100 \mu \mathrm{m}$ (Sadava, Heller et al. 2009) and secrete intercellular signals such as cytokines and chemokines across concentration gradient lengths of approximately $250 \mu \mathrm{m}$ (Francis and Palsson 1997). Compared to the characteristic lengths of non-microfluidic platforms that are generated on a scale of millimeters $(\mathrm{mm})$ to centimeters $(\mathrm{cm})$, microfluidic gradient generators can offer higher gradient resolutions and provides well-controlled hydrodynamic and mass transport conditions. Sub-micron to micrometer sized channels within microfluidic gradient generators provide concentration gradients with small length resolutions.

A microfluidic gradient generator manipulates small volumes of reagents, usually in nanolitres or microlitres, through networks of sub-micron to micrometer sized channels. The advances in microfabrication technology led to the continuous miniaturization of channel geometries, thereby reducing gradient generating lengths down to micrometer or even nanometer. The miniaturization of channel dimensions directly correlates with the reduction of the time required to transport specific concentration doses to cells, providing physiologically meaningful time scales. Microfluidic gradient generators also enable the integration and manipulation of multiple design spaces, predominately: time, chemical species and chemical concentration, into a single platform (Keenan and Folch 2008; Toetsch, Olwell et al. 2009; Chung and Choo 2010; Gupta, Kim et al. 2010). Precise control of these design spaces revolutionized the way of controlling cell microenvironments for in-vitro studies. The benefits of the minute time and length scales in microfluidics translate into precise control of concentration gradients for drug development (Mosadegh, Saadi et al. 2008; Maguire, Novik et al. 2009; Sugiura, Hattori et al. 2010; Nandagopal, Wu et al. 2011; Chen, Wo et al. 2012) and chemotaxis studies (Ahmed, Shimizu et al. 2010). Microfluidic gradient generators have also emerged as an efficient platform for controlling biomolecular microenvironments for cell culture viability studies (El-Ali, Sorger et al. 2006; Gomez-Sjoberg, Leyrat et al. 2007; Cheng, Yen et al. 2008; Ziolkowska, Jedrych et al. 2010) and stem cell differentiation (Park, Hwang et al. 2007; Gupta, Kim et al. 2010).

As microfluidic gradient generators continue to emerge as competent tools for biological studies, fundamental understanding of the transport processes would allow biologists to leverage on microfluidics to interrogate complex biological systems. In the last fifteen years, the use of microfluidic gradient generators has had a great impact on biological applications, prompting researchers in both the microfluidics and biological arena to publish several review articles (Weibel and Whitesides 2006; Keenan and Folch 2008; Kim, Kim et al. 2010; Mark, Haeberle et al. 2010; Velve-Casquillas, Le Berre et al. 2010; Beta and Bodenschatz 2011). However, most of these reviews only highlighted key biological applications of microfluidic gradient generators and placed limited emphases on the engineering aspect of microfluidics-based gradient generation. In the present review, we provide a blueprint on how users could effectively engineer concentration gradients for biological applications. The review will cover three main areas, namely: microfluidics-based mass transport phenomena, gradient generator 
designs for specific biological applications, and, a perspective on the evolution of gradient generators for biological applications.

\section{Basic mass transport phenomena in microfluidic gradient generators}

The flow field that carries concentration gradients within microfluidic devices is governed by the Navier-Stokes (N-S) equation (see Table I). The N-S equation assumes that the fluids used are incompressible and Newtonian in nature. The assumption of incompressibility is made as liquids experience negligible volume changes in response to pressure changes (Incropera, DeWitt et al. 2006). Similarly, solutions of reagents used in gradient generators are usually Newtonian and do not have viscosity values that vary with the hydrodynamic shear. Examples of Newtonian fluids used in gradient generators include water-based fluorescent dyes (Morel, Galas et al. 2012), dilute cell medium (Park, Kim et al. 2009; Kilian, Bugarija et al. 2010), dilute solutions of growth factors (Wang, Saadi et al. 2004; Mosadegh, Saadi et al. 2008), proteins (Lin, Nguyen et al. 2004; Nandagopal, Wu et al. 2011) and medical drugs (Walsh, Babin et al. 2009; Wang, Yue et al. 2009; Dai, Zheng et al. 2010). Applicable to incompressible Newtonian fluids, the constitutive N-S equation balances the rate of change in fluid momentum with the convective, viscous, and pressure forces, and other externally applied forces (see Equation 1 in Table I). In the absence of externally applied forces such as electrical or gravitational forces- as in the case of microfluidic gradient generators, the force term, $\vec{F}$, is negligible and is omitted from the N-S equation.

Table I Overview of the use of the Navier-Stokes (N-S) equation in microfluidics

Complete N-S equation $\quad \rho \frac{\partial \vec{u}}{\partial t}=-\rho \vec{u} \cdot \nabla \vec{u}+\mu \nabla^{2} \vec{u}-\nabla P+\vec{F}$ (1)
(incompressible flow)
$\begin{aligned} & \text { Where } \rho \text { is the density of the fluid, } \vec{u} \text { denotes the } \\ & \text { velocity vector of the fluid, } \mu \text { is the dynamic } \\ & \text { viscosity of the liquid, } \nabla P \text { is the pressure gradient, } \\ & \text { and } \vec{F} \text { is the external force applied to the fluid. }\end{aligned}$

Stokes equation in microfluidics

(low Reynolds number and transient

$\rho \frac{\partial \vec{u}}{\partial t}=\mu \nabla^{2} \vec{u}-\nabla P(2)$

flow)

Steady Stokes equation in microfluidics

(low Reynolds number and steady-state

$\nabla P=\mu \nabla^{2} \vec{u}$

flow)

In general, fluid flow in microchannels is laminar due to small channel dimensions. The laminar flow within microfluidic devices is characterized by Reynold's number, $R e$, that is often much smaller than $2.3 \times 10^{3}$ (Squires and Quake 2005). The Reynolds number is a dimensionless number that measures the 
relative importance between inertial $\left(\rho U^{2}\right)$ and viscous forces $\left(\frac{\mu U}{L}\right)$ and is defined as

$$
R e=\left(\rho U^{2}\right) /\left(\frac{\mu U}{L}\right)=\frac{\rho U L}{\mu}
$$

where $\rho$ is the fluid density, $U$ is the mean fluid velocity and $L$ is the characteristic length of the fluidic channel. $U$ is determined from the inlet flow rate using the volumetric flow rate equation, $Q=U A$, where $A$ is the channel cross-sectional area. In rectangular microchannels, the characteristic length $L$ is approximated as the hydraulic diameter, $D_{h}=\frac{4 * A}{S}$, where $S$ is the wetted perimeter of the channel.

Although the Reynolds number is frequently associated with fluid dynamics and mechanics, the Reynolds number has little relevance in the analysis of transport phenomena in microfluidic applications. In the microfluidic domain, the small length scales and low flow rates used result in Reynolds numbers less than 100 (Squires and Quake 2005). Mass transport analysis uses Reynolds number as an indication of the order of magnitude of flow velocities. In pure diffusion based microfluidic gradient generators with $R e<<1$, the viscous forces $\left(\mu \nabla^{2} \vec{u}\right)$ are dominant over convective forces $(\rho \vec{u} \cdot \nabla \vec{u})$ and the convective term is omitted from the N-S equation (see Equation 2 in Table I- microfluidics (low $R e$ ) N-S equation) (Deen 1998). Further simplification of the N-S equation can lead to the Stokes equation representing steady-state, low $R e$ flow presented in equations 2 and 3 of Table I.

Fluid flow velocities within microfluidic gradient generators are commonly solved by applying appropriate assumptions and simplifications to the N-S equations. These simplifications reduce the time of numerical computation or even allow an analytical solution. The velocity field forms the basis for solving mass transport problems in microfluidic gradient generators. In the next section, we introduce an analytical solution to the N-S equation with the simplification of a laminar and uni-directional flow, within pressure driven gradient generators.

\section{Mass transport in pressure driven gradient generators}

In pressure driven gradient generators, the steady state $\mathrm{N}-\mathrm{S}$ equation is commonly used to define flow velocities for a known pressure gradient $(\nabla P)$ across channel lengths, in the absence of externally applied body forces, i.e. $\vec{F}=0$. The N-S equation can be analytically solved in one direction for gradient generators that have only one non-zero velocity component, i.e. unidirectional flow. By assuming fully developed fluid flow in long microchannels, the steady state N-S equation can be solved along the channel length (in the $x$ - direction as shown in Figure 1) to give an exact solution for the average flow velocity, $U$, or flow rate, $Q$ for a known pressure drop, Equation (5). The pressure drop along the channel length occurs along the flow direction in the $x$ - axis, i.e. $\frac{\mathrm{dP}}{\mathrm{dx}}$. The velocity profile, $u$ within a rectangular microchannel varies along the $\mathrm{y}$ and $\mathrm{z}$ direction, i.e. $u=u(y, z)$. The corresponding velocity profile and channel dimensions are illustrated in Figure 1. By implementing the no-slip boundary conditions at the walls of the 
microchannel, and applying finite Fourier transformation on equation 5, an exact solution showing a linear relationship between $\Delta P$ and $Q$ can be derived, Equation (6). A detailed description of the derivations required to solve for equation 6 can be found in many textbooks (N.T. and S. 2002; Nguyen 2012). This is one of the few exact solutions of the N-S equation that can be derived analytically. Equation (6) is commonly termed as the Hagen-Poiseuille equation in many text books (N.T. and S. 2002; Kirby 2010) and defines the hydraulic resistance $\left(R_{f}\right)$ of fluid flow within a microchannel. The hydraulic resistance $R_{f}$ is dependent on the dynamic viscosity of the fluid, $\mu$, and the geometry of the microchannel.

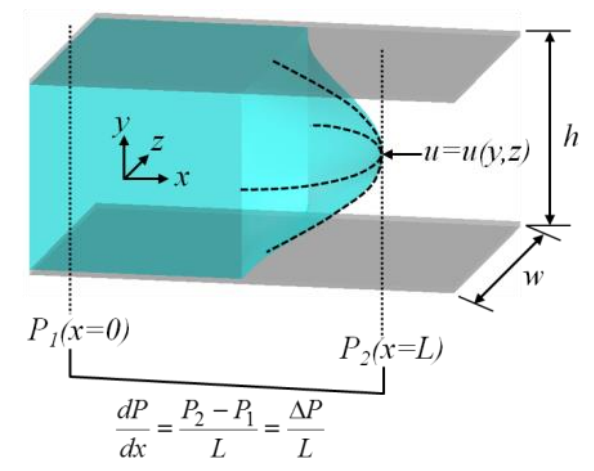

Figure 1 Fully developed laminar flow within a microchannel with a parabolic flow profile. Flow direction is from left to right and confined within the rectangular wall.

$$
\begin{gathered}
\left(\frac{\partial^{2} u}{\partial y^{2}}+\frac{\partial^{2} u}{\partial z^{2}}\right)=\frac{1}{\mu}\left(\frac{\Delta P}{L}\right) \\
\Delta P=R_{f} Q
\end{gathered}
$$

where

$$
R_{f}=\left[\frac{12 \mu L}{w h^{3}}\left\{1-\sum_{i=1,3,5, \ldots}^{\infty} \frac{192 h}{i^{5} \pi^{5} w} \tanh \left(\frac{i \pi h}{2 w}\right)\right\}^{-1}\right] \quad \text { for } \quad \text { rectangular }
$$

microchannels. In cases where the channel aspect ratio, $\frac{h}{w} \rightarrow 0, R_{f} \approx$ $\frac{12 \mu L}{w h^{3}}\left[(1-0.63 h)^{-1}\right]($ N.T. and S. 2002).

The Hagen-Poiseuille equation is commonly used to simplify fluid flow in microfluidic networks into equivalent hydraulic circuits, analogous to electric circuits (see Table II). An electric circuit can be compared to a hydraulic circuit, where the electrical voltage, resistance and current, are respectively analogous to the hydraulic pressure drop, resistance and mass flow rate. Hydraulic circuits have been used in the direct evaluation of concentration profiles in microfluidic gradient generators with sequential mixer networks, such as the "Christmas tree" design (Chung, Flanagan et al. 2005). Table II shows an example of the use of the electrical-hydraulic resistance analogy in "Christmas tree" gradient generators. As illustrated in Table II, the fluidic networks that result in fluidic resistance are analogous to an electrical network. In a fluidic network (see Table II), a concentration gradient is formed by sequential dilution of known input concentrations $\left(C_{0}\right.$ and $\left.C_{1}\right)$ through a network of mixers, followed by fluidic lamination of adjacent concentration streams into a common reservoir. With such 
designs, the flow resistances of the horizontal channels are assumed to be negligible as compared to the vertical mixing channels, and the vertical channel lengths are designed to be adequately long for complete mixing of the two input concentrations. (Dertinger, Chiu et al. 2001). With these assumptions, the resultant concentration of the solutes at the output streams is a factor of the downstream flow resistances, a concept termed here as concentration division (see Table II). At each node, the flow rate splitting of the solute and diluent streams is determined by the relative hydraulic resistances downstream from the node. This is analyzed in a similar manner as current division in electrical circuits. Although concentration division is only shown for nodes 1 to 3 in Table II, it can be applied to any of the 15 nodes within the gradient generator to determine the output concentrations from each mixer module.

Table II Electric- hydraulic analogy and its application in microfluidic gradient generators

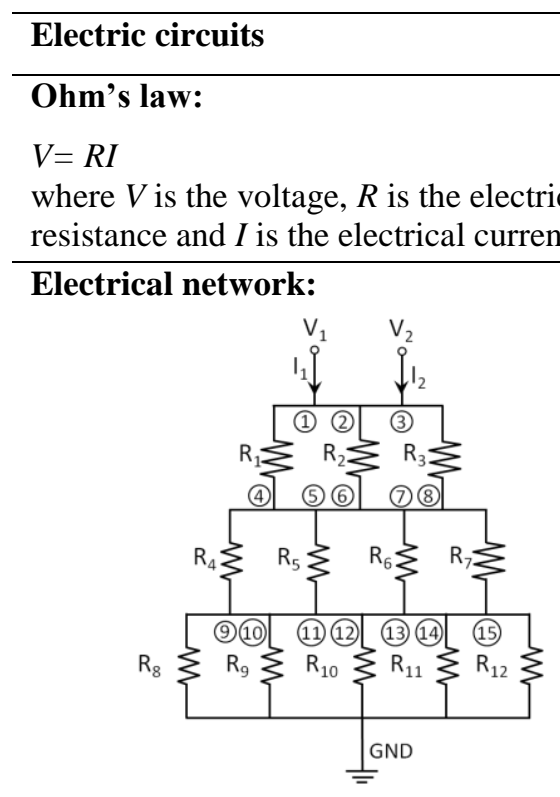

Encircled numbers represent current nodes.

\section{Hydraulic circuits}

\section{Hagen-Poiseuille equation:}

$\Delta P=R_{f} Q$

where $\Delta \mathrm{P}$ is the pressure drop, $\mathrm{R}_{\mathrm{f}}$ is the hydraulic resistance and $\mathrm{Q}$ is the flowrate.

\section{Fluidic mixer network:}

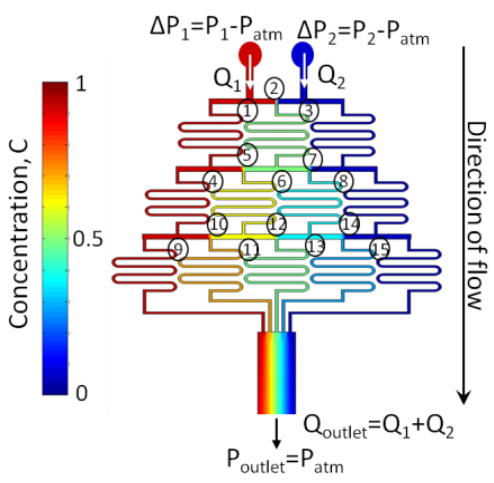

Encircled numbers represent concentration nodes.

\section{Kirchoff current law:}

At each node:

Node n: $\sum_{i=1}^{n} I_{i}=0$ where $\mathrm{n}$ is the total number of branches into or out of the node.

\section{Mass conservation:}

At each node:

Node n: $\sum_{i=1}^{n} Q_{i}=0$ where $\mathrm{n}$ is the total number of channels into or out of the node.

\section{Current division:}

Applied at nodes 1, 2 and 3:

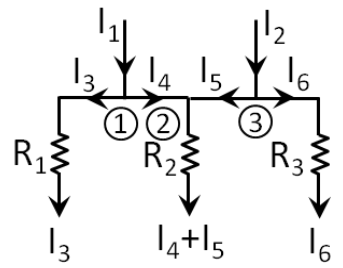

Node 1: $I_{3}=\left(\frac{R_{2}}{R_{1}+R_{2}}\right) I_{1} ; I_{4}=\left(\frac{R_{1}}{R_{1}+R_{2}}\right) I_{1}$ Node 3: $I_{5}=\left(\frac{R_{3}}{R_{2}+R_{3}}\right) I_{2} ; I_{6}=\left(\frac{R_{2}}{R_{2}+R_{3}}\right) I_{2}$

\section{Concentration division:}

Applied at nodes 1, 2 and 3:

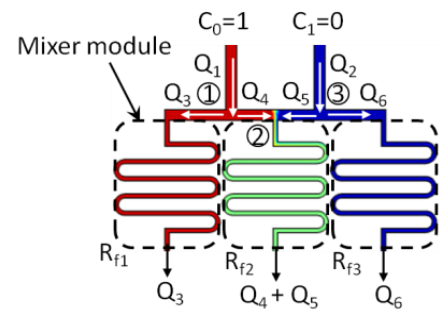

Node 1: $Q_{3}=\left(\frac{R_{f 2}}{R_{f 1}+R_{f 2}}\right) Q_{1} ; Q_{4}=\left(\frac{R_{f 1}}{R_{f 1}+R_{f 2}}\right) Q_{1}$ 


\begin{tabular}{cc} 
Node 2: $I_{4}+I_{5}=$ \\
$\left(\frac{R_{1}}{R_{1}+R_{2}}\right) I_{1}+\left(\frac{R_{3}}{R_{2}+R_{3}}\right) I_{2} \quad \begin{array}{c}\text { Node 3: } Q_{5}=\left(\frac{R_{f 3}}{R_{f 2}+R_{f 3}}\right) Q_{2} ; Q_{6}=\left(\frac{R_{f 2}}{R_{f 2}+R_{f 3}}\right) Q_{2} \\
\text { Node 2: } Q_{4}+Q_{5}=\left(\frac{R_{f 1}}{R_{f 1}+R_{f 2}}\right) Q_{1}+\left(\frac{R_{f 3}}{R_{f 2}+R_{f 3}}\right) Q_{2} \\
\text { Concentration flowing out of mixer module } R_{f 2}: \\
C_{R_{f 2}}=\frac{Q_{4} \cdot C_{0}+Q_{5} \cdot C_{1}}{\left(Q_{4}+Q_{5}\right)} ; \\
\text { Concentration out of any mixer module is: } \\
\quad C_{\text {mixer module }}=\frac{\sum_{i=1}^{2} Q_{i} \cdot C_{i}}{\sum_{i=1}^{2} Q_{i}} \\
\text { where i refers to the left and right concentration } \\
\text { streams flowing into each mixer module. }\end{array}$ \\
\hline
\end{tabular}

One of the key benefits of analytical hydraulic circuits is the ease in which channel dimensions (hydraulic resistance) can be designed to yield user-specified output concentrations. The equivalent circuit presents a particularly easy method to design the gradient generator without the use of numerical stimulation tools or prior flow experiments. Although Table II illustrates the use of hydraulic circuit analysis that is specific to the "Christmas tree" gradient generator, such circuits can also be applied in the analysis of other designs of pressure-driven gradient generators (Park, Hwang et al. 2007; Chau, Rolfe et al. 2011; Atencia, Cooksey et al. 2012). In most gradient generator designs involving pressure driven unidirectional fluid flow, the transport of solute concentrations can be simplified and analyzed as equivalent hydraulic circuits (Oh, Lee et al. 2012).

\section{Mass transport in convection/ diffusion based gradient generators}

The previous section shows how hydraulic circuit analysis is useful in determining the concentration value of individual output streams in gradient generators such as the "Christmas tree" design. However, hydraulic circuit analysis does not account for the movement of solute species across adjacent output streams and does not describe the transport of solute species in the absence of pressure driven flow. The transport of a solute species within gradient generators occurs through diffusion and/or mass convection. This process is governed by the convection-diffusion (C-D) equation that superimposes the independent processes of convection and diffusion transport of mass species. By applying the conservation of mass to a control volume, the C-D equation for a solute species balances the rate of mass accumulation with the sum of convective mass flux $\nabla \cdot(\vec{u} C)$ diffusive mass flux $D \nabla^{2} C$ and the net rate of the species generation $R_{\mathrm{g}}$ (see equation 7 in Table III). The velocity vector from the convective term, $\nabla \cdot(\vec{u} C)$, is obtained from solving the previously introduced N-S equation for convective transport of solute species ( see Table I). The net rate of species formation, $R_{g}$, is usually present when there is a chemical reaction producing or consuming the solute species. This factor is omitted in the mass transport analysis of gradient generators as negligible chemical reactivity between the solute and diluting species is assumed. This assumption is valid in gradient formation for biological studies as most solutes- growth factors, cytokines or organic compounds- have negligible chemical reactivity with their diluents. The C-D equation also accounts for diffusion as the secondary mode of mass transport. Diffusion of a single solute species in a diluent species is characterized by the 
diffusion coefficient $D$ as described by Fick's first law of diffusion (Ahmed, Shimizu et al. 2010). Fick's second law describes how mass transport via diffusion is non-steady and changes with time (Ahmed, Shimizu et al. 2010). Central to the design of diffusion dominated gradient generators, Fick's first and second laws convey two general concepts on the diffusion process in the microfluidic domain. First, concentration gradients diffuse from regions of high concentration to regions of low concentration. And second, diffusion is a time evolving phenomenon which results in an equilibration between high concentration and low concentration regions.

Table III Overview of the use of convection-diffusion (C-D) equation in microfluidic-based mass transport

\begin{tabular}{|c|c|}
\hline $\begin{array}{l}\text { Complete C-D equation } \\
\text { (binary diffusion within dilute mixtures) }\end{array}$ & $\frac{\partial C}{\partial t}+\nabla \cdot(\vec{u} C)=D \nabla^{2} C+R_{g}(7)$ \\
\hline C-D equation in Microfluidics with large & \\
\hline $\begin{array}{l}\text { Peclet number } \\
(P e ́>>1 \text { and steady-state flow) }\end{array}$ & $\nabla \cdot(\vec{u} C)=0(8)$ \\
\hline $\begin{array}{l}\text { C-D equation in Microfluidics with small } \\
\text { Peclet number } \\
(P e ́<<1 \text { and transient flow })\end{array}$ & $\frac{\partial C}{\partial t}=D \nabla^{2} C$ \\
\hline
\end{tabular}

To analyze mass transport in gradient generators, it is often necessary to use the simple form of C-D equation to describe the operational flow characteristics of the device. The simplification is usually performed via the analysis of the Péclet number (Pé). The $P e ́$ number is a measure of the relative importance of convection to diffusion (Deen 1998). It is defined as the ratio between the convective transport, $U C$ and the diffusive transport, $\frac{C}{L}$, Equation (10).

$$
P e ́=U C / D \frac{C}{L}=\frac{U L}{D}
$$

Similar to the Reynolds number Re, the Pé number is often used as an indication of the relative order of magnitude between convective and diffusive transport processes. The $P e$ number varies with the molecular diffusitivity, $D$, of the chemical species and there is more than a single $P e ́$ number in the analysis of mass transport for multiple chemical species. Within the context of microfluidic gradient generators, the $P e ́$ number is generally used to simplify the analysis of the convection-diffusion equation. When $P e ́>>1$, the dominant mode of mass transport is convection and thus the diffusive mass transport term $\left(D \nabla^{2} C\right)$ is omitted from the C-D equation. The C-D equation is further simplified when the steady-state assumption is applied to solve for the concentration field, Equation (8). When $P e ́<<1$, the dominant mode of mass transport is diffusion and hence the convective mass transport term $(\nabla \cdot(\vec{u} C))$ is omitted from the C-D equation. In cases where convective and diffusive transport mechanisms are equally important, the C-D equation has to be solved in its full form by inserting the fluid properties and boundary/ initial conditions. Simplifications to the C-D equation can be 
achieved through appropriate reductions of the problem in terms of dimensions and/or directions (Deen 1998). Analytical solutions to the C-D equation, with different forms of simplifications applied, are frequently found in many mass transport textbooks (Deen 1998; Ahmed, Shimizu et al. 2010; Beta and Bodenschatz 2011). However, in complex and multi-dimensional C-D transport problems, numerical methods are commonly applied to compute the velocity and concentration fields formed within microfluidic gradient generators. Although not a focus of this paper, the reader may refer to review papers (Boy, Gibou et al. 2008; Harting, Kunert et al. 2010) and textbooks (Velve-Casquillas, Le Berre et al. 2010) on different numerical tools used in the analysis of microfluidics- based devices.

\section{Shear stresses associated with mass transport in convection-based gradient generators}

In convection-based gradient generators, the concentration gradient depends on the flow field. During the biological experiments, the presence of a flow field introduces shear stresses to biological cells residing on the bed of the fluidic channel. As such, convection-based gradient generators have to be designed to provide cells with a physiologically relevant shear flow microenvironment, as well as provide user-specified gradients of chemical stimuli. The maximum shear stress, $\tau_{\max }$ occurs at the microchannel walls and can be determined from the parallel-plate flow approximation (Deen 1998), Equation (11).

$$
\tau_{\max }=\frac{6 \mu Q}{w h^{2}}
$$

where $\tau_{\text {wall }}$ is the shear stress induced by the flow rate, $Q$, within a microchannel of height, $h$, and width, $w, \mu$ is the dynamic viscosity of the fluid flowing through the microchannel.

The velocity and dimensional parameters used in equation 11 is illustrated in Figure 2(a). Equation 11 defines the linear and proportional relationship between shear stress and flow rate (i.e. an increase in flow rate results in a proportional increase in the shear stress induced on cells). The equation provides a reasonable estimate of the maximum shear stress applied on cells for cases where the height of microchannel, $h$, is at least one order of magnitude smaller than the microchannel width, $w$ (i.e. $\frac{h}{w}<0.1$ ). Figure $2(\mathrm{~b})$ shows the shear stress variation as channel height and flow rate are varied, for a constant channel width, $w$, of $1000 \mu \mathrm{m}$. Figure 2(b) indicates that an increase in microchannel height by one order of magnitude $(10 \mu \mathrm{m}$ to $100 \mu \mathrm{m})$ significantly reduces the shear stress applied on cells over a wide range of flow rates. As such, the selection of flow rates and microchannel geometry for gradient generation should be carefully considered in order to produce physiologically relevant cell shear environments. 
(a)

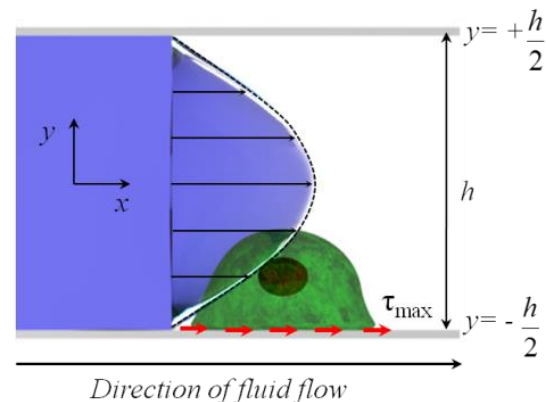

(b)

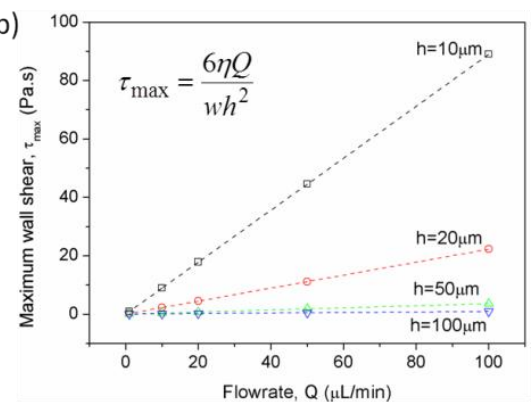

Figure 2 Shear stress in a microchannel: (a) Parallel plate flow induced shear stress on cells residing on the bed of a microchannel. (b) Linear and proportional relationship between shear stress and flowrate. Change in maximum shear stress applied on cells when microchannel height, $2 h$ is varied, for a fixed microchannel width of $w=1000 \mu \mathrm{m}$. The shear stress is plotted for water viscosity, $\mu=8.9 \times 10^{-4} \mathrm{~Pa} \cdot \mathrm{s}$.

\section{Time scales associated with mass transport in gradient generators}

The previous sections introduced the basic mass transport concepts that are pervasive in the analysis of concentration gradient formation in microfluidic devices. In those concepts, the analysis of the steady-state velocity and concentration fields include time as an implicit variable. However, in an actual gradient generation experiment, the time required to reach a steady-state or pseudo-steady state, as in the case of diffusion gradient generators, is finite and of importance. In biological experiments, it is often important to have an indication of the time required to establish the concentration gradient- the characteristic time. The characteristic time provides an order of magnitude estimate of the time required for chemicals to form a concentration gradient across a given distance. The time scale of gradient generation is also important for determining the protocol, i.e "how" the biological experiment will be carried out. For instance, in cases where the time required for gradient stability is much longer than the response time of the cell under investigation, the cell would have to be introduced into the microfluidic device after the specified concentration gradient is formed. This prevents a convolution of cell response data- the cell response should be solely influenced by the known gradient state, without any influence from unknown and transient gradient states. In Table IV, the transport parameters and hence, characteristic time scales for different types of gradient generators are summarized. The categorization of whether a gradient generator is diffusion or convection depends on the Pé number.

Table IV Overview of the characteristic time scales for microfluidic gradient generators

\begin{tabular}{ccc}
\hline Gradient generator & Dominant Transport Mode & Characteristic time scales \\
\hline Convection-based $(P e ́>1)$ & Convection & $t_{v}=\frac{L_{c}}{U}$ \\
Diffusion-based $(P e ́<1)$ & Diffusion & $t_{d}=\frac{L_{c}^{2}}{2 D}$ \\
\hline
\end{tabular}

The characteristic time scale of a microfluidic gradient generator is determined by the transport parameter and the characteristic length of the device. The transport 
parameter for convection-based gradient generators is the average flow velocity, $U$ and the transport parameter for diffusion-based gradient generators is the diffusion coefficient, $D$, respectively. The characteristic length, $L_{c}$, in time scale analysis is frequently chosen as the distance in which the concentration gradient forms. Table $\mathrm{V}$ summarizes the typical operation characteristics of some convection-based and diffusion-based gradient generators used in biological studies such as chemotaxis assays (Jeon, Baskaran et al. 2002; Lin, Nguyen et al. 2004; Abhyankar, Toepke et al. 2008; Kothapalli, van Veen et al. 2011), migration/ wound-healing assays (Wang, Saadi et al. 2004; Haessler, Kalinin et al. 2009; Haessler, Pisano et al. 2011), cytotoxicity assays (Bang, Lim et al. 2004; Ziolkowska, Jedrych et al. 2010) and cell culture (Hung, Lee et al. 2005; Cimetta, Cannizzaro et al. 2010). The specific designs of convection-based and diffusionbased gradient generators are briefly described in Table $\mathrm{V}$ and explained in detail in the later section on gradient generator designs (Figure 3).

Table V Typical operation characteristics- flow velocity $(U)$, characteristic length $\left(L_{c}\right)$, Péclet number $(P e ́)$ and characteristic time $\left(t_{c}\right)$ for gradient stabilization in convection and diffusion based gradient generators

\begin{tabular}{|c|c|c|c|c|c|c|}
\hline Reference & Type & $\begin{array}{l}\mathrm{U} \\
\left(\mathrm{mm} \mathrm{s}^{-1}\right)\end{array}$ & $\begin{array}{l}\mathrm{L}_{\mathrm{c}} \\
(\mu \mathrm{m})\end{array}$ & Chemical species & $P \dot{P e}$ & $t_{c}$ (secs) \\
\hline $\begin{array}{l}\text { (Lin, Nguyen et } \\
\text { al. 2004) }\end{array}$ & $\begin{array}{l}\text { "Christmas tree"; } \\
\text { Convective }\end{array}$ & 0.38 & 350 & $\begin{array}{l}\text { Interleukin-8 } \\
\left(\mathrm{D}=0.2-2.8 \times 10^{-12} \mathrm{~m}^{2} \mathrm{~s}^{-1}\right)\end{array}$ & $\begin{array}{l}48- \\
665\end{array}$ & 0.92 \\
\hline $\begin{array}{l}\text { (Jeon, Baskaran } \\
\text { et al. 2002) }\end{array}$ & $\begin{array}{l}\text { "Christmas tree"; } \\
\text { Convective }\end{array}$ & 1 & 500 & $\begin{array}{l}\text { Interleukin-8 } \\
\left(\mathrm{D}=2.5 \times 10^{-10} \mathrm{~m}^{2} \mathrm{~s}^{-1}\right)\end{array}$ & 2000 & 0.5 \\
\hline $\begin{array}{l}\text { (Walker, Sai et } \\
\text { al. 2005) }\end{array}$ & $\begin{array}{l}\text { "Christmas tree"; } \\
\text { Convective }\end{array}$ & $0.33,6.67$ & 500 & $\begin{array}{l}\text { FITC- dextran } \\
\left(\mathrm{D}=8 \times 10^{-11} \mathrm{~m}^{2} \mathrm{~s}^{-1}\right)\end{array}$ & $\begin{array}{l}2063 \\
4168 \\
8\end{array}$ & $\begin{array}{l}1.51 \\
0.07\end{array}$ \\
\hline $\begin{array}{l}\text { (Walsh, Babin et } \\
\text { al. 2009) }\end{array}$ & $\begin{array}{l}\text { T-junction; } \\
\text { Convective }\end{array}$ & 1.7 & 350 & $\begin{array}{l}\text { Doxorubicin } \\
\left(\mathrm{D}=2.5 \times 10^{-11} \mathrm{~m}^{2} \mathrm{~s}^{-1}\right)\end{array}$ & $\begin{array}{l}2380 \\
0\end{array}$ & 0.2 \\
\hline $\begin{array}{l}\text { (Long and Ford } \\
2009 \text { ) }\end{array}$ & $\begin{array}{l}\text { T-junction; } \\
\text { Convective }\end{array}$ & $\mathrm{n} / \mathrm{r}$ & $\mathrm{n} / \mathrm{r}$ & $\begin{array}{l}\alpha \text { - Methylasparate } \\
\left(\mathrm{D}=8.6 \times 10^{-10} \mathrm{~m}^{2} \mathrm{~s}^{-1}\right)\end{array}$ & $\begin{array}{l}20 \text { to } \\
154\end{array}$ & $\mathrm{n} / \mathrm{r}$ \\
\hline $\begin{array}{l}\text { (Park, Hwang et } \\
\text { al. 2007) }\end{array}$ & $\begin{array}{l}\text { Y-junction; } \\
\text { Convective }\end{array}$ & $\begin{array}{l}0.22 \times 10^{-3} \\
7.25 \times 10^{-3}\end{array}$ & 250 & $\begin{array}{l}\text { Polyethylene Glycol } \\
\left(\mathrm{D}=5 \times 10^{-10} \mathrm{~m}^{2} \mathrm{~s}^{-1}\right)\end{array}$ & $\begin{array}{l}3.63 \\
10.9\end{array}$ & $\begin{array}{l}1.14, \\
0.03\end{array}$ \\
\hline $\begin{array}{l}\text { (Qasaimeh, } \\
\text { Gervais et al. } \\
\text { 2011) }\end{array}$ & $\begin{array}{l}\text { 2D pressure } \\
\text { balance; } \\
\text { Convective }\end{array}$ & 2 & $\mathrm{n} / \mathrm{r}$ & $\begin{array}{l}\text { Fluorescien sodium } \\
\left(\mathrm{D}=5 \times 10^{-10} \mathrm{~m}^{2} \mathrm{~s}^{-1}\right)\end{array}$ & 5602 & 0.4 \\
\hline $\begin{array}{l}\text { (Atencia, } \\
\text { Morrow et al. } \\
2009)\end{array}$ & $\begin{array}{l}\text { 2D pressure } \\
\text { balance; Diffusive }\end{array}$ & $\mathrm{n} / \mathrm{a}$ & 1500 & $\begin{array}{l}\text { Carboxyflourescien } \\
\left(\mathrm{D}=5 \times 10^{-10} \mathrm{~m}^{2} \mathrm{~s}^{-1}\right)\end{array}$ & $\mathrm{n} / \mathrm{a}$ & $2.3 \times 10^{3}$ \\
\hline $\begin{array}{l}\text { (Du, Shim et al. } \\
\text { 2009) }\end{array}$ & $\begin{array}{l}\text { 1D flow balance; } \\
\text { Diffusive }\end{array}$ & $\mathrm{n} / \mathrm{a}$ & 50000 & $\begin{array}{l}\text { FITC- dextran } \\
\left(\mathrm{D}=1.7 \times 10^{-10} \mathrm{~m}^{2} \mathrm{~s}^{-1}\right)\end{array}$ & $\mathrm{n} / \mathrm{a}$ & $7.4 \times 10^{6}$ \\
\hline $\begin{array}{l}\text { (Ahmed, } \\
\text { Shimizu et al. } \\
\text { 2010) }\end{array}$ & $\begin{array}{l}\text { 1D gradient in } \\
\text { hydrogel; } \\
\text { Diffusive }\end{array}$ & $\mathrm{n} / \mathrm{a}$ & 1000 & $\begin{array}{l}\text { Fluorescien sodium } \\
\left(\mathrm{D}=5 \times 10^{-10} \mathrm{~m}^{2} \mathrm{~s}^{-1}\right)\end{array}$ & $\mathrm{n} / \mathrm{a}$ & $1 \times 10^{3}$ \\
\hline $\begin{array}{l}\text { (Ma, Zhou et al. } \\
\text { 2010) }\end{array}$ & $\begin{array}{l}\text { 1D gradient in 3D } \\
\text { porous scaffold; } \\
\text { Diffusive }\end{array}$ & $\mathrm{n} / \mathrm{a}$ & 1000 & $\begin{array}{l}20 \% \quad \text { Fetal Bovine } \\
\text { Serum (FBS) } \\
\left(\mathrm{D}=2.35 \times 10^{-11} \mathrm{~m}^{2} \mathrm{~s}^{-1}\right)\end{array}$ & $\mathrm{n} / \mathrm{a}$ & $2.1 \times 10^{4}$ \\
\hline $\begin{array}{l}\text { (Kothapalli, van } \\
\text { Veen et al. } \\
2011)\end{array}$ & $\begin{array}{l}2 \mathrm{D} \text { gradient in } \\
\text { collagen gel; } \\
\text { Diffusive }\end{array}$ & $\mathrm{n} / \mathrm{a}$ & 900 & $\begin{array}{l}\text { Neurite-repellant slit-2 } \\
\left(\mathrm{D}=5.1 \times 10^{-11} \mathrm{~m}^{2} \mathrm{~s}^{-1}\right)\end{array}$ & $\mathrm{n} / \mathrm{a}$ & $7.9 \times 10^{3}$ \\
\hline $\begin{array}{l}\text { (Abhyankar, } \\
\text { Toepke et al. } \\
2008)\end{array}$ & $\begin{array}{l}\text { 3D gradient in } \\
\text { agarose gel; } \\
\text { Diffusive }\end{array}$ & $\mathrm{n} / \mathrm{a}$ & 20000 & $\begin{array}{l}\text { Epidermal growth } \\
\text { factor (EGF) } \\
\left(\mathrm{D}=3.5 \times 10^{-10} \mathrm{~m}^{2} \mathrm{~s}^{-1}\right)\end{array}$ & $\mathrm{n} / \mathrm{a}$ & $5.7 \times 10^{7}$ \\
\hline $\begin{array}{l}\text { (Haessler, } \\
\text { Kalinin et al. } \\
2009)\end{array}$ & $\begin{array}{l}\text { 3D gradient in } \\
\text { collagen matrix; } \\
\text { Diffusive }\end{array}$ & $\mathrm{n} / \mathrm{a}$ & 400 & $\begin{array}{l}\text { CCL19 chemokine } \\
\left(\mathrm{D}=1.11 \times 10^{-10} \mathrm{~m}^{2} \mathrm{~s}^{-1}\right)\end{array}$ & $\mathrm{n} / \mathrm{a}$ & 720.7 \\
\hline
\end{tabular}

n/r: not reported; $n / a:$ not applicable 
Time scale analysis is important in determining the mass transport mechanism used for gradient generation. Most application specific gradient generators select mass transport modes that produce the most physiological accurate gradients for the cells being studied. The different transport modes result in vastly different characteristic times due to the range of values of the transport parameters. During microfluidic gradient generation, the typical flow velocities range from $10^{-4}$ to $10^{-}$ ${ }^{2} \mathrm{~m} / \mathrm{s}$ while typical liquid diffusion coefficients range from $10^{-8}$ to $10^{-11} \mathrm{~m}^{2} / \mathrm{s}$. Table $\mathrm{V}$ indicates that diffusive mechanisms are several orders of magnitude slower than convective mechanisms. As such, convection-based gradient generators are widely adopted for dynamic control of concentration gradients as tuning of flow rates directly control the spatial delivery of concentration gradients. On the other hand, diffusion-based gradient generators are predominantly used in producing static concentration gradients. In addition, diffusive concentration gradients can be maintained at a pseudo-steady state for time intervals on the order of several minutes to hours. Small diffusitivity, $D$, of low molecular weight chemical species in microchannels imply that concentration gradients do not change significantly over a short duration. By leveraging on this pseudo-steady state, a known concentration gradient can be sustained through periodic, rather than continuous, fluid replenishment. Diffusion-based gradient generators are thus cost-effective platforms for performing biological experiments that involve expensive reagents and/or suffer from a limited availability of reagent volume.

From an engineering perspective, characteristic time scales also help in determining the appropriate temporal resolutions of fluidic control devices. In general, most microfluidic gradient generators rely on a fluidic control devices to generate and maintain concentration gradients for biological experiments. Fluidic control tools such as syringe pumps, pressure pumps, peristaltic pumps and/or valves are commonly used in controlling fluid flow within gradient generators. Each of these flow control tools have temporal resolutions that affect the minimum response time for fluid flow control. Therefore, decisions on tool selection should be based on the intended time required for gradient generation. For instance, in convection-based generators, on-chip integrated fluid control such as membrane microvalves/ micropumps- rather than external counterparts- are frequently used to ensure minimum response lag in dynamic gradient generation (Du, Shim et al. 2009; Dai, Zheng et al. 2010; Chen, Wo et al. 2012; Frank and Tay 2013). On-chip microvalves and micropumps have extremely low dead volumes and can be integrated in the vicinity of the gradient generation chambers, thereby reducing flow response lag caused by external flow control tools. On the other end of the spectrum, diffusion-based generators transport gradients across larger time scales and seldom require rapid on-chip flow control. Many diffusionbased gradient generators utilize slow and passive tools to deliver concentration gradients. These include mechanisms such as electro-osmotic pumping (Park, Kim et al. 2009) and passive pumping (Abhyankar, Toepke et al. 2008; Berthier, Surfus et al. 2010; Kim and Kim 2010) of concentration gradients. The following section reviews how the previously discussed engineering aspects are used in gradient generator designs for different biological applications. 


\section{Microfluidic gradient generator designs for biological applications}

The engineering and operational aspects involved in designing gradient generators cumulate towards effectively using microfluidics for specific biological applications. Examples of such applications include: the study of cell migration and proliferation responses to chemical gradients, the observation of cell dynamics to temporal chemical gradients and the observation of cell responses to chemical gradients diffusing in a three-dimensional microenvironment. In the following sections, the use of different gradient designs for such biological applications is discussed.

\section{Convection-based gradient generators for investigating cell responses to chemical stimuli}

Convection-based $(P e ́>1)$ gradient generators are frequently used to study the responses of cells that experience flow-induced shear stress in their native, in-vivo microenvironments. One of the most popular designs for convection-based gradient generators is based on the integration of multiple mixer networks (previously introduced as the "Christmas tree" design) (Jeon, Baskaran et al. 2002). The design, first developed by Jeon et al., was used to generate a concentration gradient of hydrofluoric (HF) acid to etch a glass coverslip to depths proportional to the HF concentration gradients (Jeon, Dertinger et al. 2000). "Christmas tree" designs have since been used to study a plethora of biological phenomena due to its elegance in performing concentration dosing and gradient generation in a single device. The serial mixer networks within the "Christmas tree" design allow a single concentration of the solute to be serially diluted, into a range of concentration doses. The concentration doses that travel into the output chamber subsequently form 1D lateral gradients perpendicular to the laminar flow streams [see Figure 3(a) left]. In biological experiments, the interrogated cells are inserted into the output chamber where the concentration gradient is formed. "Christmas tree" gradient generators have been used to study the dosing effect of chemical stimuli to adherent cells such as MCF-7 cells (Ruan, Wang et al. 2009), human neural stem cells (Chung, Flanagan et al. 2005), MDAMB-231 breast cancer cells (Saadi, Wang et al. 2006) and human neutrophils (Lin, Nguyen et al. 2004). Recently, "Christmas tree" gradient generators have been used in the study of motile, non-adherent bacteria suspensions (Englert, Manson et al. 2009; Englert, Manson et al. 2010), representing a paradigm shift in its application. "Christmas tree" gradient generators have predominately been used in studies related to adherent cells (Jeon, Baskaran et al. 2002; Lin, Nguyen et al. 2004; Wang, Saadi et al. 2004). A study using "Christmas tree" gradient generators found that cell migration could be biased by the flow field induced by the flow system and affect cell responses to concentration gradients (Walker, Sai et al. 2005). This bias would be exacerbated in the analysis of motile cells. However, a recent study on motile E.coli bacteria utilizing "Christmas tree" gradient generation proposed that an additional control experiment, with reversed gradient directions could be used to eliminate any flow-based bias in chemotactic responses (Englert, Manson et al. 2010). 


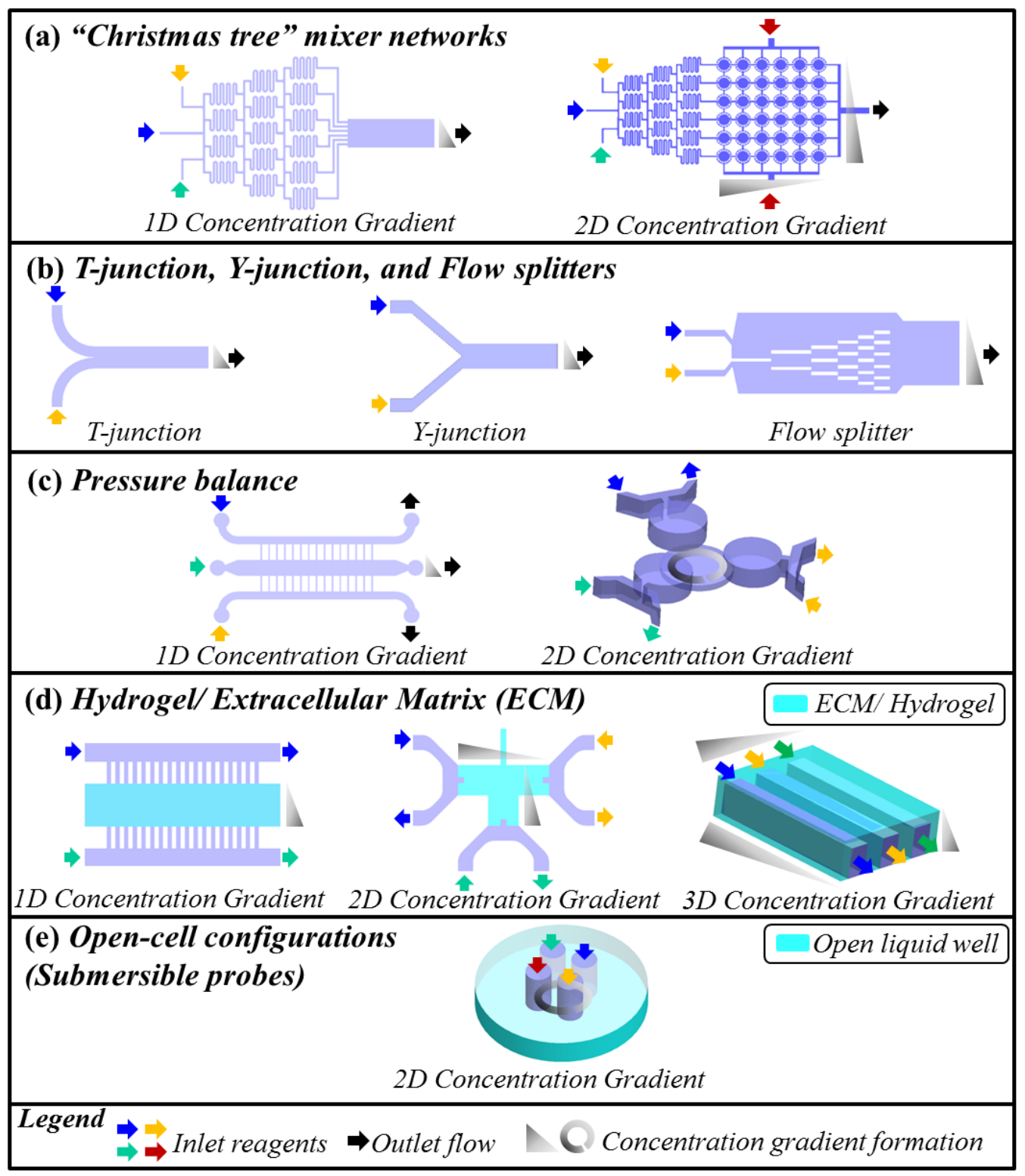

Figure 3 Microfluidics based gradient generator designs: (a) (left) "Christmas tree" design for 1D (Jeon, Dertinger et al. 2000) and (right) 2D gradient generation (Hung, Lee et al. 2005); (b) (left) Y- junction, (centre) T-junction (Long and Ford 2009) and (right) flow splitter designs (Irimia, Geba et al. 2006); (c) (left) 1D gradient generation (Irimia, Charras et al. 2007) and (right) 2D gradient generation through pressure balance designs (Atencia, Cooksey et al. 2012); (d) Molded gels for (left) 1D (Ahmed, Shimizu et al. 2010), (centre) 2D (Kothapalli, van Veen et al. 2011) and (right) 3D gradient generation (Haessler, Pisano et al. 2011), and (e) Submersible microfluidic probes (Qasaimeh, Gervais et al. 2011).

"Christmas tree" gradient generators have also been used in the field of genomics, specifically the genotyping of single nucleotide polymorphisms (SNPs). In a recent study, the generator's ability to provide temporally stable chemical gradients was applied to the discrimination of SNPs within target DNA oligonucleotides (Russom, Irimia et al. 2009). The gradient generator was used to produce the required concentration gradients of either urea or formamide. Through fluorescent detection of the allele specific probe intensity during exposure of urea or formamide gradients, the degree and speed of target DNA 
denaturisation (and associated decay of fluorescent intensity) was observed. The rate of DNA denaturisation was directly correlated to the presence of a SNP in the target DNA. By using gradient generators to produce precise doses of urea or formamide, the denaturisation of DNA duplexes could be analysed chemically instead of thermally- abrogating the need for heating control elements that are frequently used in melting curve analysis of DNA. In addition to 1D gradient generation, the "Christmas tree" design has also been employed in a highthroughput manner to produce 2D concentration gradients in cell culture microwells (Hung, Lee et al. 2005; Lee, Hung et al. 2006). Figure 3(a) (right) depicts how 1D gradient generation can be translated into a 2D gradient format in the "Christmas tree" network design.

Apart from "Christmas tree" gradient generators, other convection-based gradient generators have been reported. These generators utilize different microchannel geometries and configurations to produce concentration gradients. The more common designs include $\mathrm{Y}$-junction configurations [Figure 3(b)] to produce concentration gradients that form concentration gradients which are perpendicular to the flow field (Moore, Chou et al. 2008; Walsh, Babin et al. 2009; Meer, Vermeul et al. 2010; Atencia, Cooksey et al. 2012). Y-junction gradient generators [Figure 3(b), left] have been utilized to recreate the microenvironment surrounding tumour masses (Walsh, Babin et al. 2009). In an effort to study the effect of a chemotherapeutic drug (Doxorubicin) and anti-cancer bacteria $(S$. typhimurium) on solid tumour masses, Walsh et al. developed a multipurpose Yjunction microfluidic device. The device was used to entrap and grow tumour masses, and generate linear gradients of nutrients and drugs along the isolated tumour mass.(Walsh, Babin et al. 2009) The Y-junction generator produced a stable drug concentration gradient that could sustain for 45 hours, demonstrating the use of convection-based generators in extended biological studies. Recently, van der Meer and co-workers have also demonstrated an innovative method of performing wound healing assays through the use of a Y-junction gradient generator (Meer, Vermeul et al. 2010) [Figure 3(b), centre]. The Y-junction generator consisted of 3 input channels that merged into a main fluid stream. A confluent layer of Human umbilical vein endothelial cells (HUVECs) were first cultured on the bed of the Y-junction gradient generator. In order to conduct a wound-healing assay, a "wound" was simulated within the layer of HUVECs by applying a stream of trypsin protease. This trypsin stream was enveloped by parallel streams of cell medium. HUVECs that were exposed to the trypsin stream lost their adhesion to the channel surface and were subsequently washed away, leaving a "wound". Using this generator a growth factor $\left(\mathrm{VEGF}_{165}\right)$ gradient was supplied to study HUVEC migration vis-à-vis wound-healing (Meer, Vermeul et al. 2010). Compared to conventional wound-healing assays such as the transwell cell migration assay, the microfluidic assay demonstrated temporally stable gradients and tunable cell shear stresses that could be used to closely mimic invivo physiological conditions (Meer, Vermeul et al. 2010).

The transport dynamics of convection-based gradient generators are also extremely amenable to single cell studies. In convection-based gradient generators, flow rates in parallel concentration streams can be dynamically tuned to focus on single cells. This typically involves a Y-junction design [Figure 3(b), centre] with a minimum of 3 inlet flow channels. The flow of solute in the middle stream is enveloped by flow from parallel diluent streams that are controlled to 
focus the width of solute gradient. The width of the solute gradient can be hydrodynamically focused to single cell resolutions of several microns, by controlling the comparative flow rates of the solute and diluent streams (Nguyen 2012). Y-junction gradient generators have been used in biological experiments that test isolated cell responses to dynamic chemical stimuli (Takayama, Ostuni et al. 2003; Lucchetta, Lee et al. 2005; Rosa, Tenreiro et al. 2012; Tong, Balzer et al. 2012). Control of gradient generation on the length scale of single cells (several hundreds of microns) is simple in microchannels as the transport of concentration streams occurs in a laminar manner. Laminar flow allows the direct control of diffusion between parallel concentration streams, without the presence of chaotic advection that is dominant in macroscale flows. In a similar manner, the length scales of microfluidic gradient generators also increase the temporal resolution which is an important aspect in mimicking in-vivo biological states.

\section{Convection-based gradient generator designs for studying cell responses to pulsatile and temporal stimuli}

Convection-based gradient generators that are capable of delivering pulsatile biomolecular gradients are elegant solutions to mimicking dynamic signaling processes in cell microenvironments. In these microenvironments, pulsatile variations in biomolecular gradients regulate cell functions and are pivotal in maintaining homeostasis within a biological system. An example of this in vertebrate organisms is the homeostatic regulation of blood glucose levels by cells within the pancreas. Pancreatic islets (a group of cells within the pancreas) secrete multiple peptides, and hormones such as somatostatin, glucagon and insulin. Disrupted insulin secretion is a sign of the onset of type II diabetes mellitus and studies related to deciphering the causes of impaired insulin secretion are of clinical importance. In order to gain insight into the dynamics of in-vivo insulin secretion, convection-based gradient generators have been used to study the oscillatory release of intracellular calcium ions $\left(\mathrm{Ca}^{2+}\right)$ by pancreatic islets, in response to oscillatory glucose concentrations (Dishinger, Reid et al. 2009; Zhang, Grimley et al. 2010; Zhang, Daou et al. 2011). In-vivo, the release of intracellular $\mathrm{Ca}^{2+}$ is followed by insulin secretion and it is commonly used as an ionic marker of insulin secretion. In the report by Zhang et al., a Y-junction mixer device delivered pulsatile glucose concentrations at frequencies ranging from $0.0033 \mathrm{~Hz}$ to $0.0056 \mathrm{~Hz}$ to elicit the release of intracellular $\mathrm{Ca}^{2+}$ from the islets (Zhang, Grimley et al. 2010). Their gradient generator produced glucose concentration frequencies that were similar to in-vivo glucose oscillations. Onchip pumping frequencies and mixer channel lengths (that acted as a low-pass frequency filter) were optimized to produce glucose concentration levels that were minimally attenuated and yet, retained in-vivo pulse frequencies. Through optimization of the generator's frequency characteristics, Zhang et al. found that the release of intracellular $\mathrm{Ca}^{2+}$ by single pancreatic islets followed glucose frequencies closely, even when glucose frequencies were dramatically phaseshifted by $180^{\circ}$ in the middle of an experiment. Further studies by Zhang et al. have also yielded evidence that heterogeneous islet populations- which resemble in-vivo states even more closely than single islets- released intracellular $\mathrm{Ca}^{2+}$ in synchrony to glucose concentration frequencies (Zhang, Daou et al. 2011). In such studies, gradient generators were used to provide temporally precise concentration 
gradients for quantitative investigations of complex and dynamic biological systems.

Recently, microfluidic platforms have emerged as powerful tools for the study of the entire organism (Hulme, Shevkoplyas et al. 2010; Chung, Zhan et al. 2011) or part of tissues/ organs (Crane, Chung et al. 2010) on the size order of several millimeters. The microfluidic platforms are often fabricated with larger culture wells that are flanked by microchannels which deliver the reagents of interest at different concentration levels. These microchannels are designed to deliver concentration gradients, dynamic or otherwise, across segmented areas or the entire area of the large cell culture zone. Dynamic gradient generators have been used in "large-scale" biological studies involving worms (Dirk and Cornelia 2011), mammalian embryos (Heo, Cabrera et al. 2010), and even an entire plant organ (Meier, Lucchetta et al. 2010; Grossmann, Guo et al. 2011). In the RootChip designed by Grossmann et al., Arabidopsis thaliana roots were integrated into a microfluidic device to study root metabolism in response to pulses of 10Mm glucose (Glc) or galactose (Gal) (Grossmann, Guo et al. 2011). The Rootchip provided a platform to cultivate miniature Arabidopsis seedling roots of $\sim 5 \mathrm{~mm}$ in length, and perform subcellular observations of cystolic Glc and Gal expression during dynamic switching of Glc and Gal concentrations in the root's external environment. After performing separate experiments that exposed seedling roots to pulses of either Glc or Gal, Grossmann et al found that cystolic Glc accumulation and elimination was notably faster than that of Gal suggesting differences in the metabolic pathways of these two types of sugars (Grossmann, Guo et al. 2011).

Other gradient generators have also been designed to study the adaptation characteristics of whole organisms to dynamic chemical changes in their extracellular environment (Nagai, Ryu et al. 2010; Dirk and Cornelia 2011) In the report by Dirk and Cornelia, the behavioural dynamics of Caernorhabditis Elegans (C. Elegans) nematodes were observed in response to odor gradients and odor pulses within a microfluidic gradient generator (Dirk and Cornelia 2011). $C$. Elegans are frequently used as model organisms for animal development (Sulston 2003) and neuronal studies (Brenner 1974). Because these organisms are small ( $1 \mathrm{~mm}$ in length), microfluidic platforms that are fabricated from oxygenpermeable PDMS, serendipitously provide viable whole-animal incubation environments along with the dynamic chemical microenvironments required for biological studies. Based on the study by Dirk and Cornelia, new locomotion patterns of $C$. Elegans were observed in response to controlled temporal pulses of odor concentrations. In this study, pillar embedded incubation chambers were fabricated to resemble the native soil conditions of $C$. Elegans, aiding the generation of physiologically relevant microenvironments within the gradient generator. This aspect was lacking in earlier agarose-based studies (Dirk and Cornelia 2011). Such behavioral biology investigations on miniature organisms are increasingly being performed on microfluidic platforms. Dynamic gradient generators are expected to play a key role in recapitulating dynamic microenvironments in emerging "large scale" biological studies. 


\section{Gradient generator designs for low shear biological experiments}

Diffusion-based (with $P e ́<1$ ) gradient generators are commonly used in low shear biological studies to prevent the exposure of non-native shear stresses on cells. Cells that experience shear stresses which are not present in their native environments have been shown to respond differently to chemical gradients, as compared to native cells (Walker, Zeringue et al. 2004). To this end, diffusionbased gradient generators have been designed to leverage on the diffusive transport of chemicals in the absence of high flow velocities. In diffusion-based gradient generators, porous materials are commonly used to form concentration gradients between high concentration sources and low concentration sinks [Figure $3(\mathrm{c})$ ]. Porous materials such as hydrogels (containing extracellular matrix proteins), matrigel and agarose are frequently used in gradient generators to mimic the cellular microenvironments within the extracellular matrix. Diffusionbased gradient generators based on such materials have been tuned to contain multiple chemical species (Keenan, Hsu et al. 2006; Hamid, Blencowe et al. 2010; Suri and Schmidt 2010) and biophysical cues (Guilak, Cohen et al. 2009; Tripathi, Kathuria et al. 2009; Connelly, Gautrot et al. 2010; Kilian, Bugarija et al. 2010), thereby mimicking the in-vivo microenvironment of specific cell types. Microfluidic gradient generators containing gel materials have been designed to test a single cell population with a range of drug species or drug concentrations (Cheng, Heilman et al. 2007; Abhyankar, Toepke et al. 2008; Haessler, Kalinin et al. 2009; Ahmed, Shimizu et al. 2010; Vazquez and Paull 2010; Haessler, Pisano et al. 2011; Ricart, John et al. 2011). In these studies, gel-based gradient generators were chosen to conserve the autocrine and paracrine factors released by the cell population. Autocrine and paracrine factors may be differentially regulated at different combinatory drug concentrations and are therefore, of importance in drug development studies (Paguirigan and Beebe 2008). Gradient generators incorporating gel materials provide a platform to perform biological experiments faster and more controllably as compared to conventional gel-based assays due to precisely controlled spatial gradients.

Apart from simpler designs that involve diffusion of chemical gradients through porous gels, other microfluidic designs have recently been configured to deliver concentration gradients from multiple sources of chemical stimuli. By balancing the fluid pressure between multiple concentration sources and sinks as illustrated in Figure 3(d), overlapping diffusive gradients of multiple chemical species have been generated (Atencia, Morrow et al. 2009; Morel, Galas et al. 2012). Pressure balance (PB) within a diffusion chamber can be maintained by decoupling source flow rates around a static concentration sink. Alternate source flows at equivalent flow rates can be guided into a static concentration sink, thereby setting up a low or no shear concentration gradient environment. Conversely, PB may also be achieved along microchannels by matching the flow rates of cross flowing source and sink channels [Figure 3(d) left]. Such device configurations reduce the flow shear of the gradient forming microchannel by the destructive superposition of flow shear present in each opposing channel. PB gradient generators have been used to provide sustained chemical gradients for biological assays that lasts several hours (Cimetta, Cannizzaro et al. 2010; Brett, DeFlorio et al. 2012). Thus far, PB gradient generators have been used in low shear biological assays to study axon guidance in neurons (Queval, Ghattamaneni et al. 2010), stem cell differentiation (Park, Kim et al. 2009) and chemotaxis of highly motile non- 
adherent cells (Atencia, Morrow et al. 2009). Atencia and co-workers demonstrated the formation of a $2 \mathrm{D}$ diffusive concentration gradient through a balance of input flow pressures between three opposing flow inlets [Figure 3(d) right]. The PB scheme improves the dynamic response of diffusive gradients, as diffusive gradients often require a long time to stabilize. Dynamic chemotaxis of Pseudomonas aeruginosa was demonstrated by periodic switching of glucose sources within rotations of approximately 45 minutes (Atencia, Morrow et al. 2009). Diffusion-based generators that utilize PB schemes are attractive alternatives to convection-based generators as they allow a compromise to be reached between fast gradient stabilization and low shear flow velocities.

Furthermore, PB schemes have also been demonstrated in open chamber configurations [Figure 3(e)] that are easily amenable to biological experiments involving large tissue samples (Juncker, Schmid et al. 2005; Queval, Ghattamaneni et al. 2010; Qasaimeh, Gervais et al. 2011). Juncker et al. introduced the concept of a microfluidic probe (MFP) that generated overlapping concentration gradients by leveraging on Hele-Shaw flows between the MFP and an adjacent glass slide. During biological experiments, the cells/ tissues under investigation would be affixed onto a glass slide that was separated from the MFP by several microns. Diffusion gradients are generated across the stagnation point resulting from balancing the cross-flow of 2 injection and aspiration ports. Using the MFP, successful microperfusion of Hippoorganotypic brain slices to low shear, fluorescent dextran gradients were demonstrated in open chambers (Queval, Ghattamaneni et al. 2010). PB gradient generators are unique as they bypass the longer gradient establishment duration of diffusion-based gradient generators with hydrogel, agarose or matrigel. At the same time, PB gradient generators provide low or no shear environment for cells located at fluid stagnation wells. In addition, PB designs prevent time evolving concentration gradients that are prevalent in diffusive mass trasport through porous materials. In PB gradient generators, sources and sinks are in continuous flow, thereby preventing the depletion of molecules in the sources and the accumulation of molecules in the sinks. This circumvents the time dependence of diffusion-based mass transport in diffusion-based gradient generators with gels.

Hitherto, diffusion-based gradient generators have been widely used in low shear biological assays. While most diffusion-based gradient generators rely on gel-like materials to form diffusive gradients, new designs have been introduced to provide improved dynamic control of diffusive gradients. New designs based on pressure balance greatly improve the ability of diffusion-based generators to engineer dynamically controllable and stable gradients. However, despite the use of PB in diffusion-based generators, such generators still control concentration gradients in a slower manner than convection-based generators. To this end, another class of gradient generators has been specifically designed for low shear, yet dynamic, biological experiments. These gradient generators utilize convection-based designs to quickly generate concentration gradients and include device features that shield cells from the shear flow environment (VanDersarl, $\mathrm{Xu}$ et al. 2011; Atencia, Cooksey et al. 2012; Zheng, Wang et al. 2012). Typical designs include a high shear flow zone in which concentration gradients are generated, and a low shear flow zone in which features such as deep wells (Hung, Lee et al. 2005; Wang, Li et al. 2008) or porous membranes (VanDersarl, Xu et al. 2011) block high flow velocities but allow upstream chemical concentrations to 
diffuse to the cells. Convection-based "Christmas tree" gradient generators with deep micro-well reservoirs, as depicted in Figure 4(a), have been used to culture and shield neuronal cells from the effects of shear flow (Wang, Li et al. 2008). Neuronal cells that were seeded in the $100-\mu \mathrm{m}$ deep stagnation wells within the gradient delivery chamber experienced negligible flow shear stresses- cell responses due to chemical cues were isolated from those due to convective (shearbased) cues. Flow-shear shielding of cells within the "Christmas-tree" gradient generators has also been demonstrated through the integration of a flow restricting nano-porous membrane above the gradient chamber, Figure 4(b) (VanDersarl, Xu et al. 2011). Cells that were seeded on the nano-porous membrane experienced negligible flow shear from the underlying convective flow. Van Dersarl and coworkers reported that chemical gradients of cell staining dyes formed by the "Christmas tree" mixer networks accumulated within the cells quickly concentration gradients of stains diffused upwards and through the nano-porous membranes within 45 seconds of delivery (VanDersarl, Xu et al. 2011). These devices highlight the use of convection-based gradient generators in low shear biological experiments requiring higher temporal resolutions for gradient control.

(a)

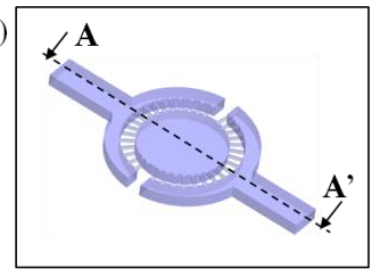

$\underline{\text { Section A-A' }}$

Flow direction

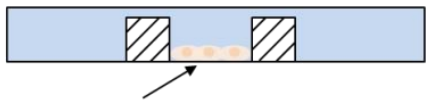

Cells seeded at the bottom of the well shielded from shear flow

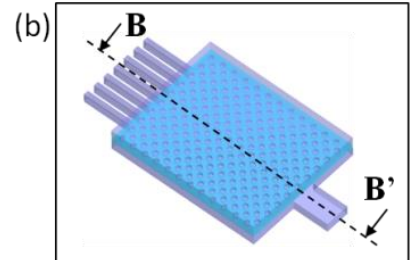

\section{Section B-B'}

Cells seeded above membrane

shielded from shear flow

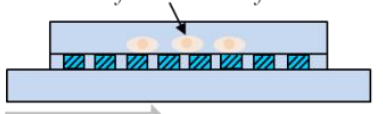

Flow direction

Legend

$\square$ Geometric features which $\quad \square$ Flow paths

shield cells from shear flow

Figure 4 Shear flow shielding by seeding cells in (a) deep microwells (Hung, Lee et al. 2005;

Wang, Li et al. 2008) or (b) on porous membranes (VanDersarl, Xu et al. 2011).

\section{Gradient generator designs for three-dimensional biological experiments}

Three-dimensional (3D) cell assays are gaining popularity in both research and clinical settings as they closely mimic in-vivo microenvironments by reconstituting cell to cell, cell to matrix and soluble factor to matrix interactions that are grossly limited under two-dimensional (2D) assay conditions (Pampaloni, Reynaud et al. 2007). These interactions are important as cell organization and function are greatly influenced by neighboring cells and, the mechanical properties and architecture of their surrounding microenvironment. Many novel materials and microfabrication techniques have been developed to move the 
current repertoire of 2D microfluidic platforms into the third dimension (Huh, Hamilton et al. 2011). Examples are 3D patternable hydrogels (Khetan and Burdick 2011), photopatternable hydrogels (Liu and Bhatia 2002) and tailored architectures of synthetic scaffolds (Greiner, Richter et al. 2012). Such materials aim to replicate the mechanical and biochemical properties of the extracellular matrix that surrounds mammalian cells under in-vivo conditions. Microfluidic platforms that are used for 3D gradient generation typically consist of cell culture domains and concentration gradient generating zones [Figure 3(c) right]. Recently, chemotaxis of dendritic cells was demonstrated on a microfluidic platform, capable of generating a 3D cell culture environment and concentration gradients of two competitive chemotactic signals, CCL21 and CCL19 (Haessler, Pisano et al. 2011). By molding fluidic conduits within porous hydrogels, as depicted in Figure 3(c) right, reagents transported within the conduits generated 3D concentration gradients within the surrounding gel. In the study by Haessler and co-workers, CCL21 and CLL19 concentrations were diffused in a 3D manner, into the surrounding hydrogel in which dendritic cells were cultured (Haessler, Pisano et al. 2011). The study highlighted differences in dendritic cell responses to $2 \mathrm{D}$ and $3 \mathrm{D}$ cell culture environments suggesting that surface and concentration gradient morphology could have a larger effect on chemotaxis assays than previously expected (Haessler, Pisano et al. 2011).

Microfluidic gradient generators have also been used as platforms for culturing cells and generating drug concentration doses for cytoxicity testing (Maguire, Novik et al. 2009; Toh, Lim et al. 2009; Sung and Shuler 2010; Wu, Huang et al. 2010; Esch, King et al. 2011). An example of this is the 3D HepaTox Chip (Toh, Lim et al. 2009) that was developed to perform several continuous assay functions such as: cell entrapment, cell culture and cell toxicity tests. Within the 3D HepaTox Chip, hepatocytes were delivered through a cell culture compartment and entrapped by surrounding micropillars. The use of micropillars enabled nutrient perfusion to confined hepatocytes, mimicking the highly permeable invivo structures of endothelial cells that separate hepatocytes from the sinusoid. In order to establish a microenvironment that is suitable for sustaining hepatocyte differentiated function, a 3D cell matrix was enveloped around the hepatocytes through laminar flow coacervation of a positively-charged modified collagen and a negatively-charged acrylate based terpolymer (Toh, Lim et al. 2009). Once sustainable cell culture conditions were created, drug toxicity studies on the 3D HepaTox Chip were performed. A linear gradient generator with bifurication channels delivered different drug doses to the hepatocytes and allowed the dosedependent cellular responses of the hepatocytes to be observed. Such gradient generator schemes allow continuous 3D cell culture and perfusion conditions while performing concentration dosing in cytotoxicity assays.

In another study, three-dimensional concentration gradients of autocrine factors were generated to improve embryonic development competence (Heo, Cabrera et al. 2010). Heo et al. utilized microchannels to perfuse media into microfunnels that were used to culture mouse embryos - peristaltic agitation of media within the microfunnels produced 3D gradients of biomolecules (autocrine factors and waste products) that were secreted by the embryos. The peristaltic transfer of media into the microfunnel was achieved by actuating Braille pins that deflected membrane valves to open or block microchannels. Results from their study showed that mimicking in-vivo ciliary currents and oviductal contractions- enabled by 
microfunnel peristaltic embryonic cultures- greatly increased embryonic development competence to that achieved by a control group of in-vivo grown embryos. The dynamic and three-dimensional architecture of this cell culture platform provided insight into how the conservation of autocrine factors, concomitant removal of secreted waste products and temporal disruption of surface concentration gradients around the embryo affect embryonic developmental competence.

\section{Conclusions and perspectives}

The development of microfluidic gradient generators will continue to evolve in resonance with the study of pertinent and ever-evolving questions in biology. New designs in gradient generator devices are largely led by the objectives of biological studies while novel microfluidic and interdisciplinary techniques will continue to lend themselves to engineering and replicating increasingly complex microenvironment cues that exist in-vivo. In this review, we introduced the major factors that influence concentration gradient generation for a wide range of biological applications. Figure 5 summarizes how the cell types and research application defines the design considerations for gradient generation. This summary can be used as a guide to selecting the type of gradient generator (convective, diffusive or pressure balanced) that best suits the research objective and the biological model to be studied.

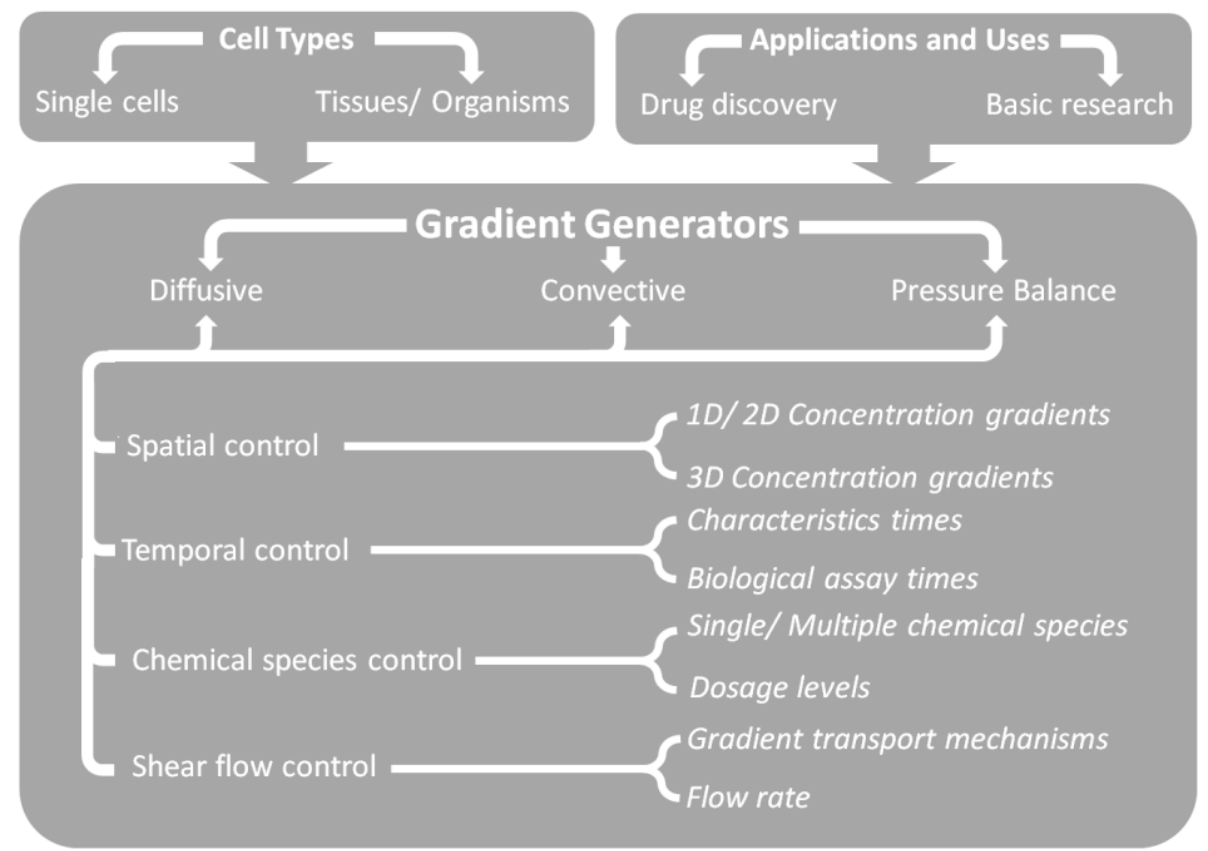

Figure 5 Design considerations for gradient generators in various biological applications.

\section{Perspectives}

The following section briefly discusses the perspective on how new developments in microfluidic gradient generators may evolve. Many gradient generators have been designed to control species concentration across the four dimensions of space and time, with limited progress in replicating the active mechanical 
regulation that exists within in-vivo microenvironments. Particularly in mammalian cell studies, the presence of dynamic substrate- induced mechanical cues have been found to affect the state of tissue cells, with likely implications on cell development, differentiation, disease and regeneration (Discher, Janmey et al. 2005). Recent developments in human-on-a-chip and organ-on-a-chip devices have demonstrated the co-culture of heterogeneous tissue cells on active and stretchable membranes that are capable of replicating in-vivo dynamic contractile and tensional forces (Esch, King et al. 2011; Huh, Leslie et al. 2012) . Although these devices are currently designed with limited and low throughput gradient generation capabilities, it is expected that the push for human-on-a-chip models to replace animal testing will see a greater need for more complex gradient generation in such devices. In human-on-a-chip models where co-culturing of different cells within a single platform is performed, gradient generators can help in efficiently generating and transporting multiple types of medium and nutrient dosages that are required to maintain the culture viability of each cell line. In line with the shift towards utilizing microfluidics in milliscale studies involving organs or tissues on a chip, it is likely that microfluidic platforms will progressively find applications in miniature whole animal or organism studies (Glaser 2011). Gradient generators can provide a controlled environment and high throughput platform to study the behavior of miniature animals to chemical signals, in a statistically reproducible manner, thereby providing insights into stimuli trigger and detection mechanisms in animal models.

From a broader perspective, it is expected that new engineering developments in microfluidic systems will move towards reducing the complexities involved in the control and execution of microfluidic-based operations. Already in the market, microfluidic-based assays have been automated for point-of-care applications such as blood tests (Samsung's IVD-A10A blood test device) and generic diagnostic testing (Lingvitae's Discipher platform and Claros's diagnostics system). Currently, most automated microfluidic platforms provide user interaction in the form of running pre-programmed biological assays with limited flexibility of performing user specified microfluidics assays, i.e. there is no system that universally automates the process of performing microfluidic experiments for basic lab research. In this area, we envision that progress in systems integration and engineering will move towards aiding uninitiated microfluidics users, in the form of "smart" microfluidic systems. "Smart" microfluidic systems receive feedback from live microscopy imaging of flow patterns and/or concentration gradients and attenuate associated flow control systems to meet user programmed flow patterns, regardless of the assay to be performed. Decoupling the assay type from basic microfluidics control would create an even greater buy-in of all microfluidic platforms, including concentration gradient generators, into biology research.

\section{References}

Abhyankar, V. V., M. W. Toepke, et al. (2008). "A platform for assessing chemotactic migration within a spatiotemporally defined 3D microenvironment." Lab on a Chip 8(9). 
Ahmed, T., T. S. Shimizu, et al. (2010). "Bacterial Chemotaxis in Linear and Nonlinear Steady Microfluidic Gradients." Nano Letters 10(9): 3379-3385.

Ahmed, T., T. S. Shimizu, et al. (2010). "Microfluidics for bacterial chemotaxis." Integrative Biology 2(11-12): 604-629.

Atencia, J., G. A. Cooksey, et al. (2012). "A robust diffusion-based gradient generator for dynamic cell assays." Lab on a Chip 12(2).

Atencia, J., J. Morrow, et al. (2009). "The microfluidic palette: A diffusive gradient generator with spatio-temporal control." Lab on a Chip 9: 2707 2714.

Bang, H., S. H. Lim, et al. (2004). "Serial dilution microchip for cytotoxicity test." Journal of Micromechanics and Microengineering 14: 1165 - 1170.

Berthier, E., J. Surfus, et al. (2010). "An arrayed high-content chemotaxis assay for patient diagnosis." Integrative Biology 2(11-12): 630-638.

Beta, C. and E. Bodenschatz (2011). "Microfluidic tools for quantitative studies of eukaryotic chemotaxis." European Journal of Cell Biology 90(10): 811816.

Boy, D. A., F. Gibou, et al. (2008). "Simulation tools for lab on a chip research: advantages, challenges, and thoughts for the future." Lab on a Chip 8(9): 1424-1431.

Boyden, S. (1962). "CHEMOTACTIC EFFECT OF MIXTURES OF ANTIBODY AND ANTIGEN ON POLYMORPHONUCLEAR LEUCOCYTES." Journal of Experimental Medicine 115(3): 453-\&.

Brenner, S. (1974). "THE GENETICS OF CAENORHABDITIS ELEGANS." Genetics 77(1): 71-94.

Brett, M.-E., R. DeFlorio, et al. (2012). "A microfluidic device that forms and redirects pheromone gradients to study chemotropism in yeast." Lab on a Chip 12(17): 3127-3134.

Chau, L. T., B. E. Rolfe, et al. (2011). "A microdevice for the creation of patent, three-dimensional endothelial cell-based microcirculatory networks." Biomicrofluidics 5(3): 034115-034114.

Chen, C.-Y., A. M. Wo, et al. (2012). "A microfluidic concentration generator for dose-response assays on ion channel pharmacology." Lab on a Chip 12(4): 794-801.

Cheng, J.-Y., M.-H. Yen, et al. (2008). "A transparent cell-culture microchamber with a variably controlled concentration gradient generator and flow field rectifier." Biomicrofluidics 2: 024105-024101 - 024105-024112.

Cheng, S.-Y., S. Heilman, et al. (2007). "A hydrogel-based microfluidic device for the studies of directed cell migration." Lab on a Chip 7(6).

Chung, B. G. and J. Choo (2010). "Microfluidic gradient platforms for controlling cellular behavior." ELECTROPHORESIS: n/a-n/a.

Chung, B. G., L. A. Flanagan, et al. (2005). "Human neural stem cell growth and differentiation in a gradient-generating microfluidic device." Lab on a Chip 5(4): 401-406.

Chung, K., M. Zhan, et al. (2011). "Microfluidic chamber arrays for wholeorganism behavior-based chemical screening." Lab on a Chip 11(21): 3689-3697.

Cimetta, E., C. Cannizzaro, et al. (2010). "Microfluidic device generating stable concentration gradients for long term cell culture: application to Wnt3a regulation of [small beta]-catenin signaling." Lab on a Chip 10(23). 
Connelly, J. T., J. E. Gautrot, et al. (2010). "Actin and serum response factor transduce physical cues from the microenvironment to regulate epidermal stem cell fate decisions." Nat Cell Biol 12(7): 711-718.

Crane, M. M., K. Chung, et al. (2010). "Microfluidics-enabled phenotyping, imaging, and screening of multicellular organisms." Lab on a Chip 10(12): 1509-1517.

Dai, W., Y. Zheng, et al. (2010). "A prototypic microfluidic platform generating stepwise concentration gradients for real-time study of cell apoptosis." Biomicrofluidics 4: 024101-024101 - 024101-024114.

Deen, W. M. (1998). Analysis of transport phenomena, Oxford University Press.

Dertinger, S. K. W., D. T. Chiu, et al. (2001). "Generation of gradients having complex shapes using microfluidic networks." Analytical Chemistry 73: 1240-1246.

Dirk, A. R. and B. I. Cornelia (2011). "High-content behavioral analysis of Caenorhabditis elegans in precise spatiotemporal chemical environments." Nature Methods 8(7): 599-605.

Discher, D. E., P. Janmey, et al. (2005). "Tissue Cells Feel and Respond to the Stiffness of Their Substrate." Science 310(5751): 1139-1143.

Dishinger, J. F., K. R. Reid, et al. (2009). "Quantitative Monitoring of Insulin Secretion from Single Islets of Langerhans in Parallel on a Microfluidic Chip." Analytical Chemistry 81(8): 3119-3127.

Du, Y., J. Shim, et al. (2009). "Rapid generation of spatially and temporally controllable long-range concentration gradients in a microfluidic device." Lab on a Chip 9(6): 761-767.

El-Ali, J., P. K. Sorger, et al. (2006). "Cells on chips." Nature 442(7101): 403411.

Englert, D. L., M. D. Manson, et al. (2009). "Flow-Based Microfluidic Device for Quantifying Bacterial Chemotaxis in Stable, Competing Gradients." Applied and Environmental Microbiology 75(13): 4557-4564.

Englert, D. L., M. D. Manson, et al. (2010). "Investigation of bacterial chemotaxis in flow-based microfluidic devices." Nature Protocols 5(5): 864-872.

Englert, D. L., M. D. Manson, et al. (2010). "Investigation of bacterial chemotaxis in flow-based microfluidic devices." Nature Protocols 5(5): 864- 872.

Esch, M. B., T. L. King, et al. (2011). "The Role of Body-on-a-Chip Devices in Drug and Toxicity Studies." Annual Review of Biomedical Engineering 13(1): 55-72.

Francis, K. and B. O. Palsson (1997). "Effective intercellular communication distances are determined by the relative time constants for cyto/chemokine secretion and diffusion." Proceedings of the National Academy of Sciences of the United States of America 94(23): 12258-12262.

Frank, T. and S. Tay (2013). "Flow-switching allows independently programmable, extremely stable, high-throughput diffusion-based gradients." Lab on a Chip.

Glaser, V. (2011) "Microfluidics making bigger impression- Techniques evolve to focus on cells, tissues and whole organisms." Genetic Engineering and Biotechnology News 31.

Gomez-Sjoberg, R., A. A. Leyrat, et al. (2007). "Versatile, fully automated, microfluidic cell culture system." Anal. Chem. 79: 8557- 8563.

Greiner, A. M., B. Richter, et al. (2012). "Micro-Engineered 3D Scaffolds for Cell Culture Studies." Macromolecular Bioscience 12(10): 1301-1314. 
Grossmann, G., W.-J. Guo, et al. (2011). "The RootChip: An Integrated Microfluidic Chip for Plant Science." The Plant Cell Online 23(12): 42344240.

Guilak, F., D. M. Cohen, et al. (2009). "Control of Stem Cell Fate by Physical Interactions with the Extracellular Matrix." Cell Stem Cell 5(1): 17-26.

Gupta, K., D. H. Kim, et al. (2010). "Lab-on-a-chip devices as an emerging platform for stem cell biology." Lab on a Chip 10(16): 2019-2031.

Haessler, U., Y. Kalinin, et al. (2009). "An agarose-based microfluidic platform with a gradient buffer for 3D chemotaxis studies." Biomedical Microdevices 11(4): 827-835.

Haessler, U., M. Pisano, et al. (2011). "Dendritic cell chemotaxis in 3D under defined chemokine gradients reveals differential response to ligands CCL21 and CCL19." Proc Natl Acad Sci USA 108(14): 5614-5619.

Haessler, U., M. Pisano, et al. (2011). "Dendritic cell chemotaxis in 3D under defined chemokine gradients reveals differential response to ligands CCL21 and CCL19." Proceedings of the National Academy of Sciences 108(14): 5614-5619.

Hamid, Z. A. A., A. Blencowe, et al. (2010). "Epoxy-amine synthesised hydrogel scaffolds for soft-tissue engineering." Biomaterials 31(25): 6454-6467.

Harting, J., C. Kunert, et al. (2010). "Lattice Boltzmann simulations in microfluidics: probing the no-slip boundary condition in hydrophobic, rough, and surface nanobubble laden microchannels." Microfluidics and nanofluidics 8(1): 1-10.

Heo, Y. S., L. M. Cabrera, et al. (2010). "Dynamic microfunnel culture enhances mouse embryo development and pregnancy rates." Human Reproduction 25(3): 613-622.

Huh, D., G. A. Hamilton, et al. (2011). "From 3D cell culture to organs-on-chips." Trends in cell biology 21(12): 745-754.

Huh, D., D. C. Leslie, et al. (2012). "A Human Disease Model of Drug ToxicityInduced Pulmonary Edema in a Lung-on-a-Chip Microdevice." Science Translational Medicine 4(159): 159ra147-159ra147.

Hulme, S. E., S. S. Shevkoplyas, et al. (2010). "Lifespan-on-a-chip: microfluidic chambers for performing lifelong observation of C. elegans." Lab on a Chip 10(5): 589-597.

Hung, P. J., P. J. Lee, et al. (2005). "Continuous perfusion microfluidic cell culture array for high-throughput cell-based assays." Biotechnology and Bioengineering 89(1): 1-8.

Incropera, F. P., D. P. DeWitt, et al. (2006). Fundamentals of Heat and Mass Transfer, John Wiley \& Sons, Inc.

Irimia, D., G. Charras, et al. (2007). "Polar stimulation and constrained cell migration in microfluidic channels." Lab on a Chip 7(12): 1783-1790.

Irimia, D., D. A. Geba, et al. (2006). "Universal microfluidic gradient generator." Analytical Chemistry 78: 3472 - 3477.

Jeon, N. J., S. K. W. Dertinger, et al. (2000). "Generation of solution and surface gradients using microfluidic systems." Langmuir 16: 8311-8316.

Jeon, N. L., H. Baskaran, et al. (2002). "Neutrophil chemotaxis in linear and complex gradients of interleukin- 8 formed in a microfabricated device." Nature Biotechnology 20: $826-830$.

Juncker, D., H. Schmid, et al. (2005). "Multipurpose microfluidic probe." Nat Mater 4(8): 622-628. 
Keenan, T. M. and A. Folch (2008). "Biomolecular gradients in cell culture systems." Lab on a Chip 8: 34-57.

Keenan, T. M., C. H. Hsu, et al. (2006). "Microfluidic "jets" for generating steady-state gradients of soluble molecules on open surfaces." Applied Physics Letters 89(11).

Khetan, S. and J. A. Burdick (2011). "Patterning hydrogels in three dimensions towards controlling cellular interactions." Soft Matter 7(3): 830-838.

Kilian, K. A., B. Bugarija, et al. (2010). "Geometric cues for directing the differentiation of mesenchymal stem cells." Proceedings of the National Academy of Sciences 107(11): 4872-4877.

Kim, M. and T. Kim (2010). "Diffusion-Based and Long-Range Concentration Gradients of Multiple Chemicals for Bacterial Chemotaxis Assays." Analytical Chemistry 82(22): 9401-9409.

Kim, S., H. J. Kim, et al. (2010). "Biological applications of microfluidic gradient devices." Integrative Biology 2(11-12): 584-603.

Kirby, B. (2010). Micro- and Nanoscale Fluid Mechanics: Transport in Microfluidic Devices, Leiden : Cambridge University Press.

Kothapalli, C. R., E. van Veen, et al. (2011). "A high-throughput microfluidic assay to study neurite response to growth factor gradients." Lab on a Chip.

Kothapalli, C. R., E. van Veen, et al. (2011). "A high-throughput microfluidic assay to study neurite response to growth factor gradients." Lab on a Chip 11(3): 497-507.

Lee, P. J., P. J. Hung, et al. (2006). "Nanoliter scale microbioreactor array for quantitative cell biology." Biotechnology and Bioengineering 94(1): 5-14.

Li, G. N., J. Liu, et al. (2008). "Multi-molecular gradients of permissive and inhibitory cues direct neurite outgrowth." Annals of Biomedical Engineering 36(6): 889-904.

Lin, F. (2009). A MICROFLUIDICS-BASED METHOD FOR ANALYZING LEUKOCYTE MIGRATION TO CHEMOATTRACTANT GRADIENTS. Methods in Enzymology, Vol 461: Chemokines, Part B. 461: 333-347.

Lin, F., C. M. C. Nguyen, et al. (2004). "Effective neutrophil chemotaxis is strongly influenced by mean IL-8 concentration." Biochemical and Biophysical Research Communications 319(2): 576-581.

Liu, V. and S. Bhatia (2002). "Three-Dimensional Photopatterning of Hydrogels Containing Living Cells." Biomedical Microdevices 4(4): 257-266.

Long, T. and R. M. Ford (2009). "Enhanced Transverse Migration of Bacteria by Chemotaxis in a Porous T-Sensor." Environmental Science \& Technology 43(5): 1546-1552.

Lucchetta, E. M., J. H. Lee, et al. (2005). "Dynamics of Drosophila embryonic patterning network perturbed in space and time using microfluidics." Nature 434(7037): 1134-1138.

Ma, L., C. Zhou, et al. (2010). "A porous 3D cell culture micro device for cell migration study." Biomedical Microdevices 12(4): 753-760.

Maguire, T. J., E. Novik, et al. (2009). "Design and application of microfluidic systems for in vitro pharmacokinetic evaluation of drug candidates." Current Drug Metabolism 10: 1192- 1199.

Mark, D., S. Haeberle, et al. (2010). "Microfluidic lab-on-a-chip platforms: requirements, characteristics and applications." Chemical Society Reviews 39(3): 1153-1182. 
Meer, A. D. v. d., K. Vermeul, et al. (2010). "A microfluidic wound-healing assay for quantifying endothelial cell migration." Am. J Physiol Heart Circ Physiol 298: H719- H725.

Meier, M., E. M. Lucchetta, et al. (2010). "Chemical stimulation of the Arabidopsis thaliana root using multi-laminar flow on a microfluidic chip." Lab on a Chip 10(16): 2147-2153.

Moore, T. I., C. S. Chou, et al. (2008). "Robust Spatial Sensing of Mating Pheromone Gradients by Yeast Cells." PLoS ONE 3(12).

Morel, M., J.-C. Galas, et al. (2012). "Concentration landscape generators for shear free dynamic chemical stimulation." Lab on a Chip 12(7).

Mosadegh, B., W. Saadi, et al. (2008). "Epidermal growth factor promotes breast cancer cell chemotaxis in CXCL12 gradients." Biotechnology and Bioengineering 100(6): 1205-1213.

N.T., N. and W. S. (2002). Fundamentals and Applications of Microfluidics. Boston, London, Artech House.

Nagai, M., S. Ryu, et al. (2010). "Chemical control of Vorticella bioactuator using microfluidics." Lab on a Chip 10(12): 1574-1578.

Nandagopal, S., D. Wu, et al. (2011). "Combinatorial Guidance by CCR7 Ligands for T Lymphocytes Migration in Co-Existing Chemokine Fields." PLoS ONE 6(3): e18183.

Nguyen, N.-T. (2012). Micromixers- Fundamentals, Design and Fabrication, Elsevier.

Oh, K. W., K. Lee, et al. (2012). "Design of pressure-driven microfluidic networks using electric circuit analogy." Lab on a Chip 12(3): 515-545.

Paguirigan, A. L. and D. J. Beebe (2008). "Microfluidics meets cell biology: bridging the gap by validation and application of microscale techniques for cell biological assays." Bioessays 30(9): 811.

Pampaloni, F., E. G. Reynaud, et al. (2007). "The third dimension bridges the gap between cell culture and live tissue." Nat Rev Mol Cell Biol 8(10): 839845.

Park, J. Y., C. M. Hwang, et al. (2007). "Gradient generation by an osmotic pump and the behavior of human mesenchymal stem cells under the fetal bovine serum concentration gradient." Lab on a Chip 7(12): 1673-1680.

Park, J. Y., S.-K. Kim, et al. (2009). "Differentiation of Neural Progenitor Cells in a Microfluidic Chip-Generated Cytokine Gradient." STEM CELLS 27(11): 2646-2654.

Qasaimeh, M. A., T. Gervais, et al. (2011). "Microfluidic quadrupole and floating concentration gradient." Nat Commun 2: 464.

Queval, A., N. R. Ghattamaneni, et al. (2010). "Chamber and microfluidic probe for microperfusion of organotypic brain slices." Lab on a Chip 10(3).

Ricart, B. G., B. John, et al. (2011). "Dendritic Cells Distinguish Individual Chemokine Signals through CCR7 and CXCR4." The Journal of Immunology 186(1): 53-61.

Rosa, P., S. Tenreiro, et al. (2012). "High-throughput study of alpha-synuclein expression in yeast using microfluidics for control of local cellular microenvironment." Biomicrofluidics 6(1).

Ruan, J., L. H. Wang, et al. (2009). "Fabrication of a microfluidic chip containing dam, weirs and gradient generator for studying cellular response to chemical modulation." Materials Science \& Engineering C-Biomimetic and Supramolecular Systems 29(3): 674-679. 
Russom, A., D. Irimia, et al. (2009). "Chemical gradient-mediated melting curve analysis for genotyping of SNPs." ELECTROPHORESIS 30(14): 25362543.

Saadi, W., S. J. Wang, et al. (2006). "A parallel-gradient microfluidic chamber for quantitative analysis of breast cancer cell chemotaxis." Biomedical Microdevices 8(2): 109-118.

Sadava, D., C. Heller, et al. (2009). Life: The science of biology, Sinauer Associates, Inc. .

Squires, T. M. and S. R. Quake (2005). "Microfluidics: Fluid physcis at the nanolitre scale." Rev. Mod. Phys. 77(3): 977- 1026.

Squires, T. M. and S. R. Quake (2005). "Microfluidics: Fluid physics at the nanoliter scale." Reviews of Modern Physics 77(3): 977-1026.

Sugiura, S., K. Hattori, et al. (2010). "Microfluidic Serial Dilution Cell-Based Assay for Analyzing Drug Dose Response over a Wide Concentration Range." Analytical Chemistry 82(19): 8278-8282.

Sulston, J. E. (2003). "Caenorhabditis elegans: The Cell Lineage and Beyond (Nobel Lecture)." ChemBioChem 4(8): 688-696.

Sung, J. H. and M. L. Shuler (2010). "In vitro microscale systems for systematic drug toxicity study." Bioprocessing and Biosystem Engineering 33: 5 - 19.

Suri, S. and C. E. Schmidt (2010). "Cell-Laden Hydrogel Constructs of Hyaluronic Acid, Collagen, and Laminin for Neural Tissue Engineering." Tissue Engineering Part A 16(5): 1703-1716.

Takayama, S., E. Ostuni, et al. (2003). "Selective Chemical Treatment of Cellular Microdomains Using Multiple Laminar Streams." Chemistry \& Biology 10(2): 123-130.

Toetsch, S., P. Olwell, et al. (2009). "The evolution of chemotaxis assays from static models to physiologically relevant platforms." Integrative Biology 1(2): 170-181.

Toh, Y.-C., T. C. Lim, et al. (2009). "A microfluidic 3D hepatocyte chip for drug toxicity testing." Lab on a Chip 9(14).

Tong, Z., E. M. Balzer, et al. (2012). "Chemotaxis of Cell Populations through Confined Spaces at Single-Cell Resolution." PLoS ONE 7(1): e29211.

Tripathi, A., N. Kathuria, et al. (2009). "Elastic and macroporous agarose-gelatin cryogels with isotropic and anisotropic porosity for tissue engineering." Journal of Biomedical Materials Research Part A 90A(3): 680-694.

VanDersarl, J. J., A. M. Xu, et al. (2011). "Rapid spatial and temporal controlled signal delivery over large cell culture areas." Lab on a Chip 11(18): 30573063.

Vazquez, M. and B. Paull (2010). "Review on recent and advanced applications of monoliths and related porous polymer gels in micro-fluidic devices." Analytica Chimica Acta 668(2): 100-113.

Velve-Casquillas, G., M. Le Berre, et al. (2010). "Microfluidic tools for cell biological research." Nano Today 5(1): 28-47.

Walker, G. M., J. Sai, et al. (2005). "Effects of flow and diffusion on chemotaxis studies in a microfabricated gradient generator." Lab on a Chip 5(6): 611618.

Walker, G. M., H. C. Zeringue, et al. (2004). "Microenvironment design considerations for cellular scale studies." Lab on a chip 4: 91-97.

Walsh, C. L., B. M. Babin, et al. (2009). "A multipurpose microfluidic device designed to mimic microenvironment gradients and develop targeted cancer therapeutics." Lab on a Chip 9(4): 545-554. 
Wang, C. J., X. Li, et al. (2008). "A microfluidics-based tuning assay reveals complex growth cone responses to integrated gradients of substrate-bound ECM molecules and diffusible guidance cues." Lab Chip 8: 227- 237.

Wang, C. J., X. Li, et al. (2008). "A microfluidics-based turning assay reveals complex growth cone responses to integrated gradients of substrate-bound ECM molecules and diffusible guidance cues." Lab on a Chip 8(2): 227237.

Wang, S. J., W. Saadi, et al. (2004). "Differential effects of EGF gradient profiles on MDA-MB-231 breast cancer cell chemotaxis." Experimental Cell Research 300(1): 180-189.

Wang, S. Y., F. Yue, et al. (2009). "Application of microfluidic gradient chip in the analysis of lung cancer chemotherapy resistance." Journal of Pharmaceutical and Biomedical Analysis 49(3): 806-810.

Weibel, D. B. and G. M. Whitesides (2006). "Applications of microfluidics in chemical biology." Current Opinion in Chemical Biology 10(6): 584-591.

Wu, M. H., S. B. Huang, et al. (2010). "Microfluidic cell culture systems for drug research." Lab on a Chip 10(8): 939-956.

Zhang, X., A. Daou, et al. (2011). "Synchronization of mouse islets of Langerhans by glucose waveforms." Am J Physiol Endocrinol Metab 301(4): E742747.

Zhang, X., A. Grimley, et al. (2010). "Microfluidic system for generation of sinusoidal glucose waveforms for entrainment of islets of Langerhans." Anal. Chem. 85(15): 6704- 6711.

Zheng, G. X., Y. H. Wang, et al. (2012). "Microalgal motility measurement microfluidic chip for toxicity assessment of heavy metals." Analytical and Bioanalytical Chemistry 404(10): 3061-3069.

Zicha, D., G. A. Dunn, et al. (1991). "A NEW DIRECT-VIEWING

CHEMOTAXIS CHAMBER." Journal of Cell Science 99: 769-775.

Zigmond, S. H. and J. G. Hirsch (1973). "Leukocyte locomotion and chemotaxis

New methods for evaluation and demostration of a cell-dervice chemotactic factor." J. Exp. Med. 137(2): 387-410.

Ziolkowska, K., E. Jedrych, et al. (2010). "PDMS/glass microfluidic cell culture system for cytotoxicity tests and cells passage." Sensors and Actuators BChemical 145(1): 533-542. 